ARTICLE

https://doi.org/10.1038/s414.67-021-23762-0

\title{
Phospholipids of APOE lipoproteins activate microglia in an isoform-specific manner in preclinical models of Alzheimer's disease
}

Nicholas F. Fitz ${ }^{1,4}$, Kyong Nyon Nam¹,4, Cody M. Wolfe (1) 1,4, Florent Letronne', Brittany E. Playso', Bistra E. Iordanova (1) ${ }^{2}$, Takashi D. Y. Kozai ${ }^{2}$, Richard J. Biedrzycki', Valerian E. Kagan (1) ${ }^{1}$, Yulia Y. Tyurina ${ }^{1}$, Xianlin Han (10 ${ }^{3}$, Iliya Lefterov ${ }^{1 凶} \&$ Radosveta Koldamova (i) ${ }^{1 凶}$

APOE and Trem2 are major genetic risk factors for Alzheimer's disease (AD), but how they affect microglia response to $A \beta$ remains unclear. Here we report an APOE isoform-specific phospholipid signature with correlation between human $A P O E \varepsilon 3 / 3$ and $A P O E \varepsilon 4 / 4$ AD brain and lipoproteins from astrocyte conditioned media of APOE3 and APOE4 mice. Using preclinical AD mouse models, we show that APOE3 lipoproteins, unlike APOE4, induce faster microglial migration towards injected $A \beta$, facilitate $A \beta$ uptake, and ameliorate $A \beta$ effects on cognition. Bulk and single-cell RNA-seq demonstrate that, compared to APOE4, cortical infusion of APOE3 lipoproteins upregulates a higher proportion of genes linked to an activated microglia response, and this trend is augmented by TREM2 deficiency. In vitro, lack of TREM2 decreases A $\beta$ uptake by APOE4-treated microglia only, suggesting TREM2-APOE interaction. Our study elucidates phenotypic and transcriptional differences in microglial response to $A \beta$ mediated by APOE3 or APOE4 lipoproteins in preclinical models of AD.

\footnotetext{
${ }^{1}$ Deparment of Environmental and Occupational Health, University of Pittsburgh, Pittsburgh, PA, USA. ${ }^{2}$ Department of Bioengineering, University of Pittsburgh, Pittsburgh, PA, USA. ${ }^{3}$ Barshop Institute for Longevity and Aging Studies, San Antonio, TX, USA. ${ }^{4}$ These authors contributed equally: Nicholas F. Fitz, Kyong Nyon Nam, Cody M. Wolfe.凶email: iliyal@pitt.edu; radak@pitt.edu
} 
ate-onset Alzheimer's disease $(\mathrm{AD})$ is age-related dementia characterized by amyloid $\beta$ (A $\beta)$ plaques, neurofibrillary tangles, and cognitive decline ${ }^{1}$. Inheritance of Apolipoprotein $(A P O E) \varepsilon 4$ allele is the major genetic risk factor for late-onset $\mathrm{AD}$, modifying disease risk and progression in an isoformdependent manner $(\varepsilon 2<\varepsilon 3<\varepsilon 4$, with $\varepsilon 4$ bringing the highest risk $)^{2,3}$. Isoform differences in amino acids at position 112 affects lipid binding properties of APOE and at 158 the receptor-binding affinity to APOE receptors ${ }^{4}$. APOE is a major lipid transporter and the structural differences result in different lipid binding properties. Thus, APOE4 preferentially binds lower density lipoproteins (LDL, VLDL) whereas APOE3 and APOE2 bind high-density lipoproteins $(\mathrm{HDL})^{5}$. In a recent study we applied shotgun lipidomics to measure the major phospholipid classes and their molecular species in brains of AD patients of different $A P O E$ genotypes $^{6}$. Our data demonstrated that few of these phospholipids were significantly lower in $A P O E \varepsilon 4 / c$ carriers vs. $A P O E \varepsilon 2 / c$ carriers $^{6}$. In the brain, APOE is primarily secreted by astrocytes and, to a lesser extent microglia, in a lipid-poor form ${ }^{7}$. ABCA1 transporter mediates the transfer of phospholipids and cholesterol to lipid-free APOE, thus forming discoidal HDL particles (reviewed in Koldamova et al. $^{8}$ ). APOE3 and APOE4 lipoproteins isolated from astrocytes conditioned media (referred to as E3 and E4 lipoproteins) are composed of proteins, cholesterol, and phospholipids and deficient of cholesteryl esters ${ }^{9}$. It has been reported that the APOE lipidation state significantly impacts $A \beta$ aggregation $^{10}$ and $A \beta$ clearance at the blood-brain barrier $(\mathrm{BBB})^{11}$. APOE4 isoform is associated with impaired $A \beta$ uptake, clearance, and degradation ${ }^{12}$, resulting in higher amyloid load in APP transgenic mice ${ }^{13,14}$. Interestingly, a study by Verghese et al. suggested that APOE influences $A \beta$ clearance in vivo not through direct binding but via its interaction with other cell surface receptors $^{15}$.

Microglia can have beneficial effects assisting $A \beta$ clearance and by forming a barrier surrounding amyloid plaques but can also be detrimental, causing chronic neuroinflammation (reviewed in Deczkowska et $\mathrm{al}^{16}$. and Hansen et $\mathrm{al}^{17}$.). Recent comprehensive single-cell RNA sequencing (scRNA-seq) analysis in mouse models of neurodegeneration discovered a subset of microglia named disease-associated microglia $(\mathrm{DAM})^{18,19}$. The upregulation of DAM genes is usually accompanied by a decreased expression of homeostatic microglia genes responsible for maintaining the normal microglia function of surveillance. Among $\mathrm{DAM}$ genes are genes associated with increased risk for $\mathrm{AD}$, such as APOE and TREM2. It has been reported that TREM2 can recognize a variety of ligands including APOE, HDL, and LDL, which could affect microglial interaction with $\mathrm{A} \beta$ and $\mathrm{APOE}^{20-22}$. APOE and TREM2 are essential for the development of microglia barrier around the plaques ${ }^{23}$. Recently, we demonstrated that the lack of TREM2 differentially affects the phenotype and transcriptome of human APOE3- and APOE4-expressing mice ${ }^{24}$. TREM2 deficiency increased plaque growth concurrently to the impairment of microglia barrier, an effect most pronounced at earlier stages of amyloid deposition. Interestingly, lack of Trem2 significantly decreased plaque-associated APOE protein only in APOE4 but not in APOE3 mice in agreement with gene expression data.

In this study, after performing lipidomic analysis of native E3and E4-lipoproteins and brain samples from $A P O E \varepsilon 3 / 3$ and APOE\&4/4 AD patients, we identified a common phospholipid signature between their major phospholipid classes. We hypothesized that isoform-specific phospholipid composition of E3 and E4 native lipoproteins would elicit distinct phenotypic and transcriptomic response by microglia. We used in vivo twophoton imaging and determined that the infusion of $\mathrm{A} \beta$ with $\mathrm{E} 3$ lipoproteins induced a more rapid microglial response than with
E4 lipoproteins. Behavioral testing following cortical $A \beta$ infusion demonstrated that both native lipoproteins ameliorated $\mathrm{A} \beta$ deleterious effects on cognition, with APOE3 exhibiting a higher efficacy than APOE4. To determine how E3 and E4 lipoproteins affect gene expression in microglia in response to $A \beta$, we performed bulk and scRNA-seq. Collectively, the data showed that the addition of $\mathrm{E} 3$ lipoproteins to $\mathrm{A} \beta$ upregulated a higher proportion of DAM genes and is associated with more active transcriptomic response than E4. scRNA-seq identified microgliaspecific clusters affected by Trem 2 deficiency, suggesting that lack of Trem2 impairs the transition of microglia from homeostatic to activating state. Compared to E3, E4-expressing microglia showed a reduced $A \beta$ uptake that was additionally aggravated by Trem 2 deficiency. Together, our findings have elucidated unique phenotypic and transcriptional differences in the microglial response to $\mathrm{A} \beta$ in the presence of $\mathrm{E} 3$ or $\mathrm{E} 4$ lipoproteins.

\section{Results}

Common APOE dependent phospholipid signature between human AD brains and native APOE lipoproteins. Previously, we have shown a significant APOE isoform-specific difference in phospholipid content in the brains of $\mathrm{AD}$ patients, specifically that $\mathrm{AD}$ brains from $A P O E \varepsilon 2$ carriers have a unique lipid profile ${ }^{6}$. In the present study, we compared the phospholipid profile of native APOE lipoproteins isolated from conditioned media of APOE3 and APOE4 primary astrocytes to those of APOE\&4/4, $A P O E \varepsilon 4$ carriers, and APOE\&3/3 brain samples from $\mathrm{AD}$ patients (Supplementary Table 1). The primary astrocytes were established from mice expressing human APOE3 or APOE4 isoforms ${ }^{24}$. To measure the most abundant nine phospholipid classes in human brain, we re-analysed multi-dimensional mass spectrometry shotgun lipidomics (MDMS-SL) data used in Lefterov et $\mathrm{al}^{6}$., and compared it with a new MDMS-SL analysis of APOE lipoproteins. We found that out of nine phospholipid classes, five (PE, PI, PS, SM, and PA) had a statistically higher level in $A P O E \varepsilon 3 / 3$ vs. $A P O E \varepsilon 4 / 4$ brains while $A P O E \varepsilon 4$ carrier levels were in the middle (Fig. 1a). Overall, except of PG, APOE-containing lipoproteins had a similar direction of fold change for each class as the human samples (Fig. 1b). In the lipoprotein dataset, we found that PI, PS, SM, and LPC were statistically higher in E3 vs. E4 lipoproteins (for the abbreviation see the legend of Fig. 1). We also found that total phospholipids concentration was higher in CSF of APP/E3 vs. APP/E4 mice (Supplementary Fig. 1k). Lipoproteins derived from E3/Abca1 ${ }^{\text {het }}$ and E4/Abca $1^{\text {het }}$ astrocytes $^{13}$ were used as negative controls and, as expected, demonstrated half of the phospholipid content of their wild-type counterparts (Supplementary Fig. 1). Correlation analysis (Fig. 1c) demonstrated that a very strong correlation existed between all phospholipid species examined, excluding PG. As shown on Fig. 1d, e, 103 lipid species were commonly identified in the human and lipoprotein samples, most of which were affected in APOE isoform-specific manner in both datasets and were higher in E3 samples.

To confirm the results from shotgun lipidomics of APOE native particles, we applied liquid chromatography-mass spectrometry (LC-MS) and measured five of the phospholipid classes reported to comprise the bulk of brain APOE-containing lipoproteins ${ }^{9}$, namely: PC, PE, PI, and PS; while CL was used as a control. The tSNE clustering shown on Fig. If demonstrated that the phospholipid molecular species from the two APOE isoforms segregated in separate groups, forming distinct clusters. Similarly to the MDMS-SL results, we found that the negatively charged phospholipids (PI, PE, and PS) were significantly higher in E3 than in E4 lipoproteins while CL was unchanged (Fig. 1g, h). Additionally, specific subspecies of PE and PS (PE36:1, PE36:2, PS36:1, and PS40:6) represented a large portion of the 

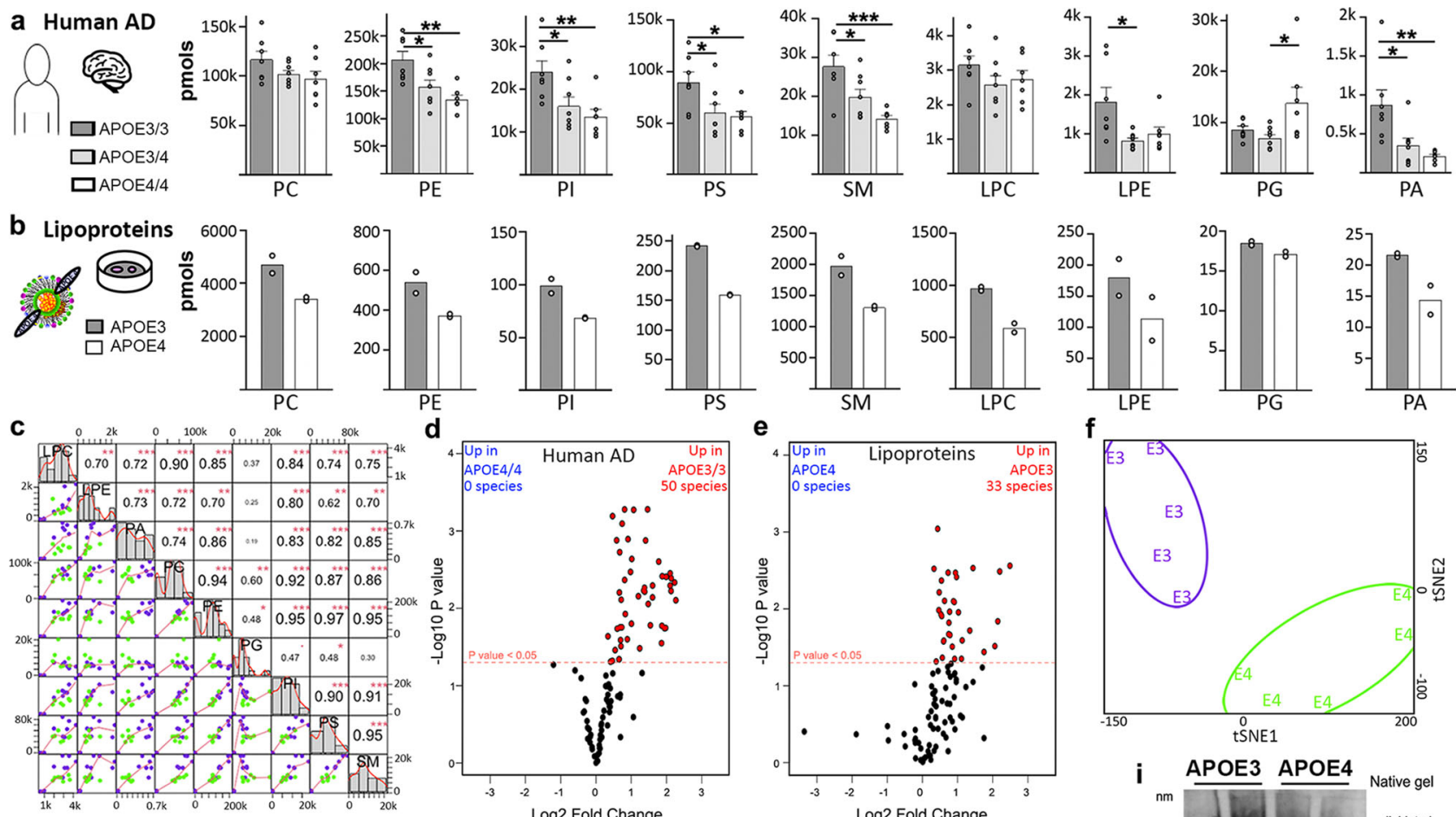

d
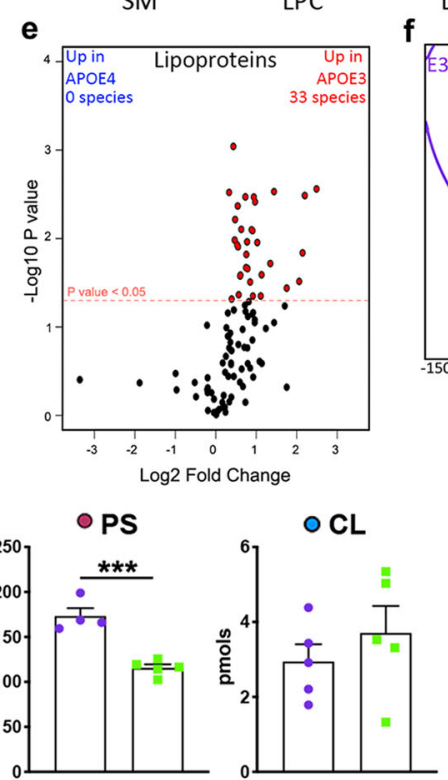

g
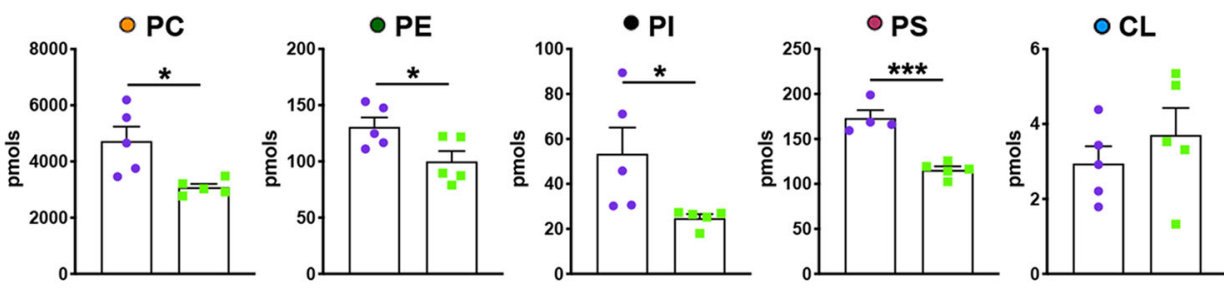

h o Phosphatidylcholine (PC)
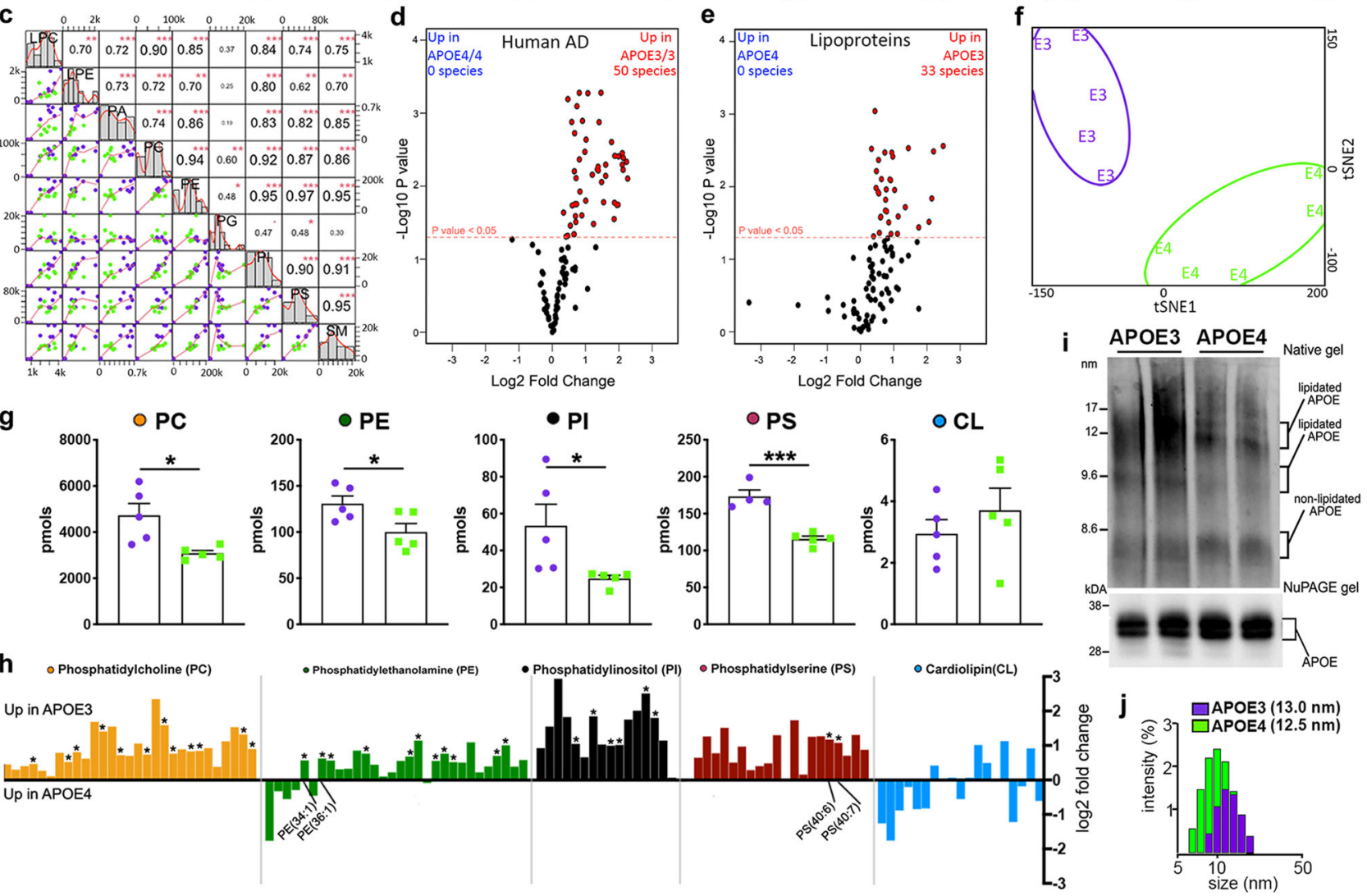

total amount of those classes and were found in significantly less abundance in APOE4 lipoproteins. These specific subspecies may represent potential lipid activation signals which impact receptor binding and could activate signal transduction pathways.

Since the diameter of lipoproteins is commonly used to represent their lipidation ${ }^{25}$, we used non-denaturing native gel electrophoresis (Fig. 1i) and dynamic light scattering assay (Fig. 1j) to determine the diameter of APOE native particles. The results demonstrated that the size of E3 native lipoproteins peaked at $13 \mathrm{~nm}$ while the size of E4 particles peaked at $12.5 \mathrm{~nm}$ (Fig. 1j). The native gels (Fig. 1i) localized APOE within three populations of particles, migrating around 12,9 , and $8 \mathrm{~nm}$. In agreement with a previous study on HDL, the measurements allowed us to conclude that particles with a diameter between 12 and $9 \mathrm{~nm}$ corresponded to discoidal $\alpha-\mathrm{HDL}$ and were lipidated, while particles with a size of $8 \mathrm{~nm}$ corresponded to pre- $\beta$-HDL and were lipid poor/non-lipidated ${ }^{26}$. SDS NuPAGE (Fig. 1i, bottom panel) confirmed that an equal amount of APOE protein was used for native gels. Lastly, Supplementary Fig. 11 shows that the cholesterol content of E3 lipoproteins was slightly but significantly increased compared to E4, with no difference in the amount of free cholesterol or cholesterol ester (Supplementary Fig. $1 \mathrm{~m})$.

APOE lipoproteins rescue $A \boldsymbol{\beta}$-induced memory impairments. Prior studies demonstrated that the injection of oligomeric $A \beta$ into brain caused significant memory deficits ${ }^{27,28}$. Here we tested the effect of native E3 and E4 lipoproteins on $\mathrm{A} \beta$ oligomerization as before ${ }^{29}$. As visible from Supplementary Fig. $2 a-c, A \beta$ incubation with either E3 or E4 native lipoproteins virtually abolished the formation of oligomers. In fact, we were able to detect $A \beta$ oligomers only when $\mathrm{A} \beta$ was incubated alone without E3 or E4. Thus, in terms of preventing $A \beta$ oligomer formation both E3 and E4 native particles seem equally effective at preventing it as determined by our detection methods. Next, to examine if the addition of native APOE lipoproteins to $A \beta$ ameliorates $A \beta$ induced memory deficits, $A \beta \pm \mathrm{APOE}$ (referred to as $\mathrm{A} \beta \mathrm{E} 3$ or $\mathrm{A} \beta \mathrm{E} 4)$ were infused into the cortex of young WT mice followed by behavioral testing as shown on Fig. $2 \mathrm{a}-\mathrm{d}$. For both tests, A $\beta$ oligomers significantly worsened cognitive performance compared to their corresponding negative control (Fig. 2b, d). In 
Fig. 1 Common phospholipid signature between APOE 8 4/4 and APOE\&3/3 human AD brains and APOE3 and E4 native lipoproteins. a, b The

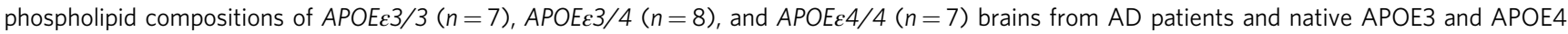
lipoproteins were determined using MDMS-SL. Brain samples were from the inferior parietal lobe. Native lipoproteins were derived from pooled APOE3 or APOE4 astrocyte conditioned media and two samples of each were measured. Bar charts of nine lipid classes analyzed by Shotgun lipidomics in human (a) and native lipoprotein (b) datasets. Analysis is by ANOVA followed by Tukey's multiple comparison test. PE, $p=0.0256$ (E33 vs. E34), $p=0.0021$ (E3/3 vs. $\mathrm{E} 4 / 4$ ); $\mathrm{Pl}, p=0.0432$ (E33 vs. E34), $p=0.0094$ (E3/3 vs. E4/4); $\mathrm{PS}, p=0.0464$ (E33 vs. E34), $p=0.0274$ (E3/3 vs. E4/4); SM, $p=0.0357$ (E33 vs. $\mathrm{E} 34), p=0.0007$ (E3/3 vs. E4/4); LPE, $p=0.0139$ (E33 vs. E34); $\mathrm{PG}, p=0.0326$ (E3/3 vs. E4/4); PA, $p=0.0177$ (E33 vs. E34), $p=0.0039$ (E3/3 vs. E4/4). c Correlation matrix of the nine lipid classes analyzed in human and lipoprotein lipidomics data shows correlation scatter plots (bottom-left), histograms of lipid amount (diagonal), and correlation coefficients between lipid classes (top-right). Purple dots denote $A P O E \varepsilon 3 / 3$, green dots denote $A P O E \varepsilon 4 / 4$. Axes, pmol for each lipid species. d, e Volcano plots depict all 103 lipid species commonly identified in the human (d) and lipoprotein (e) datasets. Red denotes species which are significantly upregulated in APOEE3/3 brains or APOE3 lipoproteins. $\mathrm{X}$ axis, log2 fold change and y axis, -log1OP value for all lipid species. e-j Data for native APOE3 or APOE4 lipoproteins derived from astrocyte conditioned media. $\mathbf{f}-\mathbf{h}$ LC/MS analysis of five Phospholipid classes of APOE3 and E4 lipoproteins. $\mathbf{f} \mathrm{tSNE}$ plot shows unique clustering of all lipids from lipoprotein samples according to APOE isoform: E3 (purple) and E4 (green). $\mathbf{g}$ Bar charts of major lipid classes of E3 and E4 native lipoproteins comprising all the lipids species shown in panel $\mathbf{h}$. Analysis by two-tailed unpaired $t$-test. PC, $p=0.0152 ; \mathrm{PE}, p=0.0390 ; \mathrm{PI}, p=0.0417 ; \mathrm{PS}, p=0.0003$. h Bar chart representing fold change of E3 compared to E4 lipoproteins for individual lipid species from five lipid classes. Significantly impacted species which are found in abundance from the PS and PE classes are labeled. i Top: Native gel electrophoresis probed for APOE. The diameter of native markers (in nm) are shown on the left. The migration of lipidated and non-lipidated APOE is shown on the right indicated with brackets. Bottom: SDS-NuPAGE gel followed by Western blot for APOE. $\mathbf{j}$ The size distribution of E3 (purple) and E4 (green) native lipoproteins was assessed by dynamic light scattering using a Zetasizer. Lipidomic analysis was performed on two independent APOE preparations measured in duplicate. The phospholipid content for each APOE isoform was normalized to APOE protein amount as determined by SDS NUPAGE. Bars represent mean \pm SEM. ${ }^{\star} p<0.05 ;{ }^{\star \star} p<0.01 ;{ }^{\star \star \star} p<0.001$. CL Cardiolipin, LPC lysophosphatidylcholine, LPE lysophosphatidylethanolamine, PA phosphatidic acid, PC phosphatidylcholine, PE phosphatidylethanolamine, PG phosphatidylglycerol, PI phosphatidylinositol, PS phosphatidylserine, and SM sphingomyelin.

contrast, injection of both $\mathrm{A} \beta \mathrm{E} 3$ or $\mathrm{A} \beta \mathrm{E} 4$ significantly ameliorated the effects of $A \beta$ on cognitive performance. Importantly, the cognitive performance in mice infused with $A \beta E 4$ remained significantly worse compared to their $A \beta E 3$ counterparts when tested by novel object recognition (NOR). For both tests, behavior controls showed no significant differences between the groups (Supplementary Fig. 2d-f). We concluded that both E3 and E4 native lipoproteins decreased $A \beta$ aggregation and ameliorated its deleterious effects on cognitive performance, with E3 demonstrating a better efficacy.

To investigate the effect of $\mathrm{A} \beta \mathrm{E} 3$ and $\mathrm{A} \beta \mathrm{E} 4$ infusions on transcriptome, we injected them into the cortex and performed RNA-seq using sorted microglia and neurons. There were no differentially expressed genes (DEGs) when comparing A $\beta E 3$ vs. $\mathrm{A} \beta \mathrm{E} 4$ neuronal transcriptomes (Fig. 2e). In contrast, there were 1792 DEGs in microglia: 722 upregulated in mice injected with $A \beta E 3$ and 1072 genes upregulated in $A \beta E 4$ injected mice (Fig. $2 \mathrm{f}$ and Supplementary Data 1). As shown on Fig. $2 \mathrm{~g}, \mathrm{f}$, upregulated in $A \beta E 3$ vs. $A \beta E 4$ microglia were many microgliaspecific genes (Tmem119, P2ry13, and Trem2) or genes associated with reactive microglia such as cytokines and cathepsins (Supplementary Data 1). Furthermore, biological processes upregulated by $\mathrm{A} \beta \mathrm{E} 3$ injections were more connected to activated microglia phenotype (GO:0002376-immune system process, GO:0031663-lipopolysaccharide-mediated signaling pathway). In contrast, the genes upregulated in $\mathrm{A} \beta \mathrm{E} 4$ injected mice and biological process associated with them were less specific to microglia and their activation (Fig. $2 \mathrm{~h}$ and Supplementary Data 1).

We also examined the morphology of microglia surrounding the injection site using IBA1 staining and Imaris filament module to trace microglial projections (Fig. 2i, j). The heatmap of microglial projections around the infusion site demonstrated that microglia in the $A \beta E 3$ group had significantly increased branch length and branch points (bar graph insets), and the projections were oriented more toward the infusion site (the area between heat maps) when compared to the $\mathrm{A} \beta \mathrm{E} 4$ group. We also performed macrophage-specific F4/80 immunohistochemistry analysis. Figure $2 \mathrm{k}-\mathrm{m}$ shows an increased F4/80/Adgre1 immunostaining around the infusion site in mice injected with $\mathrm{A} \beta \mathrm{E} 3$ compared to $\mathrm{A} \beta \mathrm{E} 4$, which is consistent with the overall reduction in Adgre1 gene expression seen in A $\beta \mathrm{E} 4$ microglia.

We also examined whether microglial barrier surrounding amyloid plaques in APP/PS1 mice depends on APOE isoform by using mice expressing human APOE3 and APOE4 (referred to as APP/E3 and APP/E4 ${ }^{24}$ ). As shown on Supplementary Fig. 3a, b, linear regression analysis demonstrates a statistically significant relationship between microglia barrier and $A \beta$ plaques in the cortex of APP/E3 mice but not in APP/E4 mice, resulting in less plaques in APP/E3 mice. This suggests that in APP/E3 mice microglia barrier increases around developing plaques, thus restricting their growth. Furthermore, Imaris tracing of microglial processes shows diminished branch number and length in APP/ E4 compared to APP/E3 mice (Supplementary Fig. 3c-f), similar to the acute effect after the $A \beta$ infusion in WT mice. Thus, the results from gene expression, microglia morphology, and microglia barrier in APP mice suggest that E3 induce stronger microglial response when compared to E4.

Microglia interaction with $\mathbf{A} \boldsymbol{\beta}$ is affected by APOE isoform. We first compared the effect of E3 and E4 lipoproteins on $A \beta$ uptake using primary microglia isolated from WT mice. $A \beta$ alone and $A \beta$ preincubated with $\mathrm{A} \beta \mathrm{E} 3^{\text {het }}$ lipoproteins were used as control. As shown on Supplementary Fig. 4a-e, labeled A $\beta$ uptake was significantly increased after co-incubation with either of the lipoproteins, but the effect of native E3 was stronger.

To determine if the presence of native E3 and E4 lipoproteins affects microglia function in vivo, we used $\mathrm{Cx} 3 \mathrm{cr} 1 \mathrm{GFP}$ mice that express EGFP protein in microglia and peripheral mononuclear cells and fluorescently-labeled A $\beta$. First, we tested the impact of lipoproteins on $A \beta$ uptake of microglia by injecting $A \beta E 3$ or $\mathrm{A} \beta \mathrm{E} 4$ into the cortex and performing flow cytometry at 4 and 24 $\mathrm{h}$ post-injection (Fig. $3 \mathrm{a}-\mathrm{c}$ and Supplementary Fig. $5 \mathrm{a}-\mathrm{c}$ ). The cell suspension was separated on microglia without $A \beta$ (green signal, single + ) and microglia that had engulfed $\mathrm{A} \beta$ (green + red signal, dual +$)$. As shown on Fig. 3b, c, E3 lipoproteins were more efficient than E4 in facilitating A $\beta$ uptake by microglia at $24 \mathrm{~h}$, but not at $4 \mathrm{~h}$ after the injection.

To further examine if APOE native lipoproteins have an impact on the microglial response to infusion of $A \beta$, we employed 


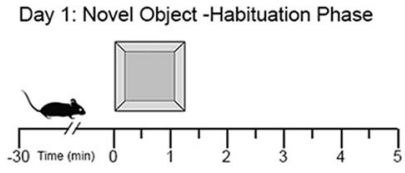

Day 2: Novel Object -Training Phase

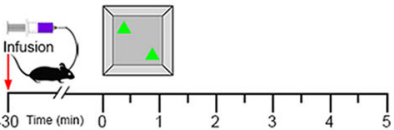

Day 3: Novel Object -Testing Phase

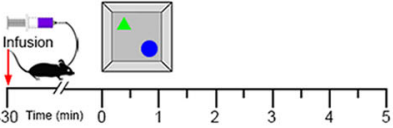

b

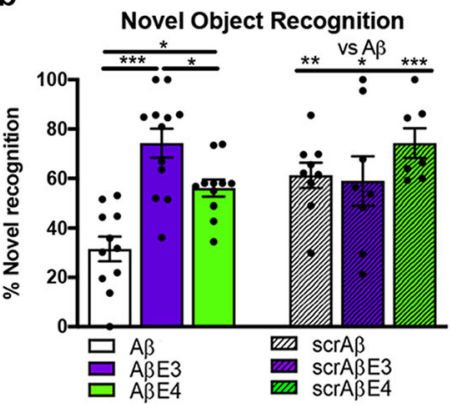

i
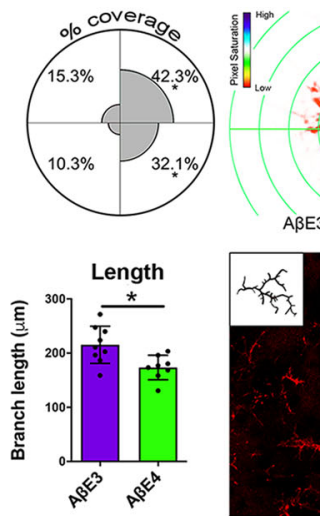

C

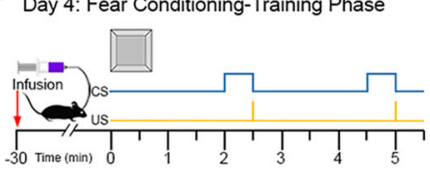

Day 5: Fear Conditioning-Contextual Phase

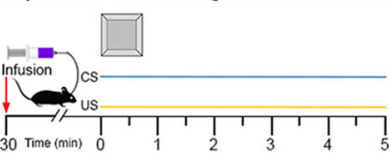

Day 6: Fear Conditioning-Cued Phase

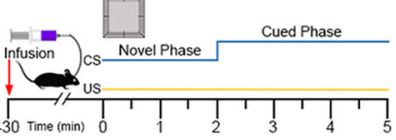

d

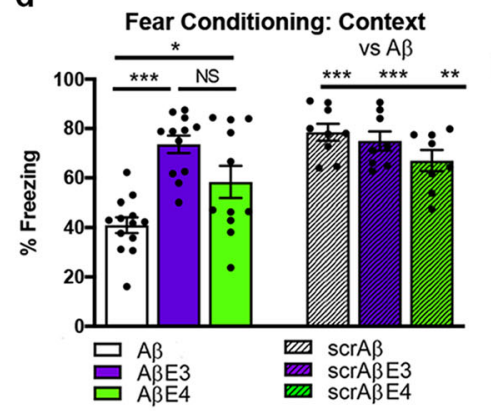

j

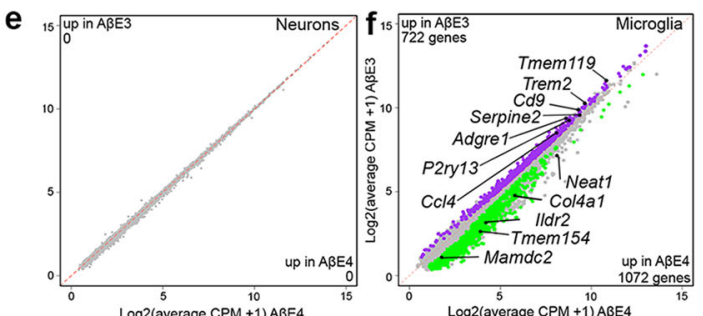

g

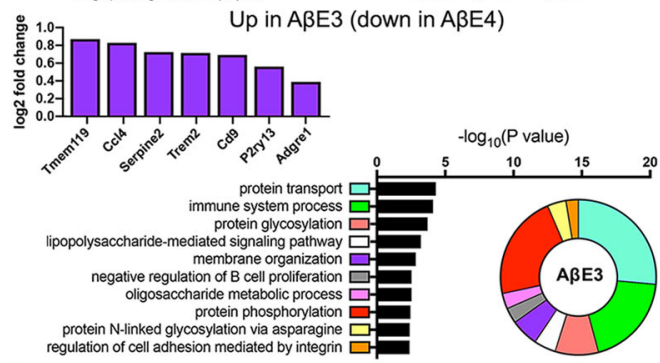

h

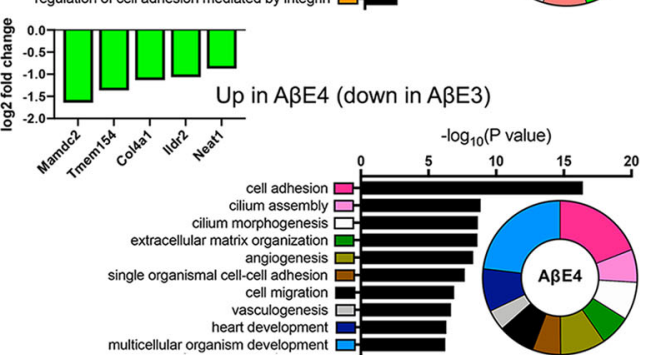

$\mathbf{k}$

nfusion Site
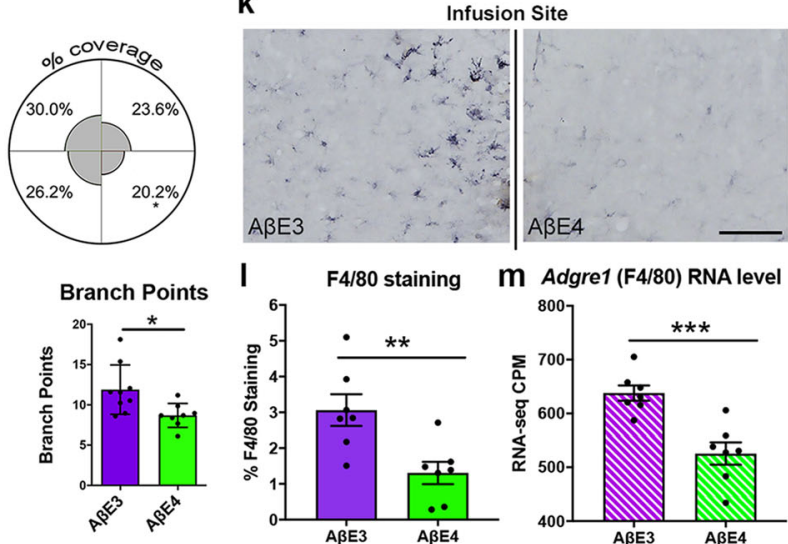

Fig. 2 Effect of native E3 and E4 lipoproteins on A $\beta$ induced cognitive decline and microglial response. a-d $A \beta$ preparations after $24 \mathrm{~h}$ incubation (A $\beta$, white; $A \beta E 3$, purple; and $A \beta E 4$, green) were infused into the brain of 2-month-old WT mice and cognitive performance examined by novel object recognition ( $\mathbf{a}, \mathbf{b})$ and fear conditioning $(\mathbf{c}, \mathbf{d})$. Scrambled $A \beta(\operatorname{scr} A \beta)$ was used as negative control ( $\operatorname{scr} A \beta+$ Veh, scr $A \beta E 3$, and scr $A \beta E 4$; line pattern). All mice were infused 30 min prior to training on days 2 through 6 of behavioral testing as shown on $\mathbf{a}$ and $\mathbf{c}$. Analysis by one-way ANOVA followed by Tukeys multiple comparison test. $A \beta$ vs. $A \beta E 3, p<0.0001 ; A \beta$ vs. $A \beta E 4, p=0.0044 ; A \beta E 3$ vs. $A \beta E 4, p=0.0351 ; A \beta$ vs. scrA $\beta, p=0.0088 ; A \beta$ vs. scr $A \beta E 3, p=$ $0.0251 ; A \beta$ vs. scr $A \beta E 4, p=0.0002$ for novel object recognition. $A \beta$ vs. $A \beta E 3, p<0.0001 ; A \beta$ vs. $A \beta E 4, p=0.0388 ; A \beta$ vs. scr $A \beta, p<0.0001 ; A \beta$ vs. $\operatorname{scr} A \beta E 3, p<0.0001 ; A \beta$ vs. scrA $\beta E 4, p=0.0015$ for fear conditioning. $n=12$ for $A \beta \pm E 3$ or $E 4$, and $n=8$ for scrA $\beta$ controls. The same $A \beta$ preparations were infused into the cortex of another group of WT mice and $24 \mathrm{~h}$ later microglia and neurons from one cortex were sorted via MACS and used for RNAseq. $n=5$ mice per group. e, $\mathbf{f}$ Scatterplots of neuronal (e) and microglial (f) gene expression profile from A $\beta E 3$ vs. A $\beta E 4$ injected. Genes significantly upregulated at FDR $<0.05$ in $A \beta E 3$ (purple), and $A \beta E 4$ (green). $\mathbf{g}$, $\mathbf{h}$ Bar plots of significantly upregulated genes and GO terms of $A \beta E 3$ ( $\mathbf{g}$, purple) and $A \beta E 4$ (h, green) groups. $\mathbf{i}, \mathbf{j}$ IBA1 staining around the infusion site of the same A $\mathrm{BE} 3$ (i, purple) or A $\beta E 4$ ( $\mathbf{j}$, green) groups. Imaris filament tracer was used for generation of a microglial projection heatmap around the infusion site. Bottom: representative images of IBA1 staining with Imaris tracing of the labeled $\left({ }^{\star}\right)$ microglia as an inset. Scale bar $=50 \mu \mathrm{m} . n=3$ mice/group; 86 microglia/A $\beta E 3$ and 106 microglia/A $\beta E 4$. Bar graphs show increased branch length and points of microglia around the infusion site of the A $\beta E 3$ (purple) vs. A $\beta E 4$ (green) group. Analysis by two-tailed unpaired $t$-test; $p=0.0103$ for branch length and $p=0.0173$ for branch points. $\mathbf{k}-\mathbf{m}$ Representative images of the $F 4 / 80$ (Adgre1) immunostaining around the infusion site of $A \beta E 3$ or $A \beta E 4$ group (k). Images are positioned with the infusion site in the middle. Scale bar $=100 \mu \mathrm{m}$. I Bar graph showing percent coverage of $\mathrm{F} 4 / 80$ staining increased around the infusion site of the A $\beta E 3$ (purple) vs. A $B$ E4 (green) group. $p=0.0069$ ( $\mathbf{m}$ ) Bar graph showing mRNA level of Adgre1 as determined by RNA-seq (CPM, count per million, line pattern, $p=0.0007$ ). Analysis by two-tailed unpaired $t$-test. $n=7$ mice per group. Bars represent mean \pm SEM. * $p<0.05,{ }^{\star \star} p<0.01 ;{ }^{\star \star \star} p<0.001$; NS no significance. 

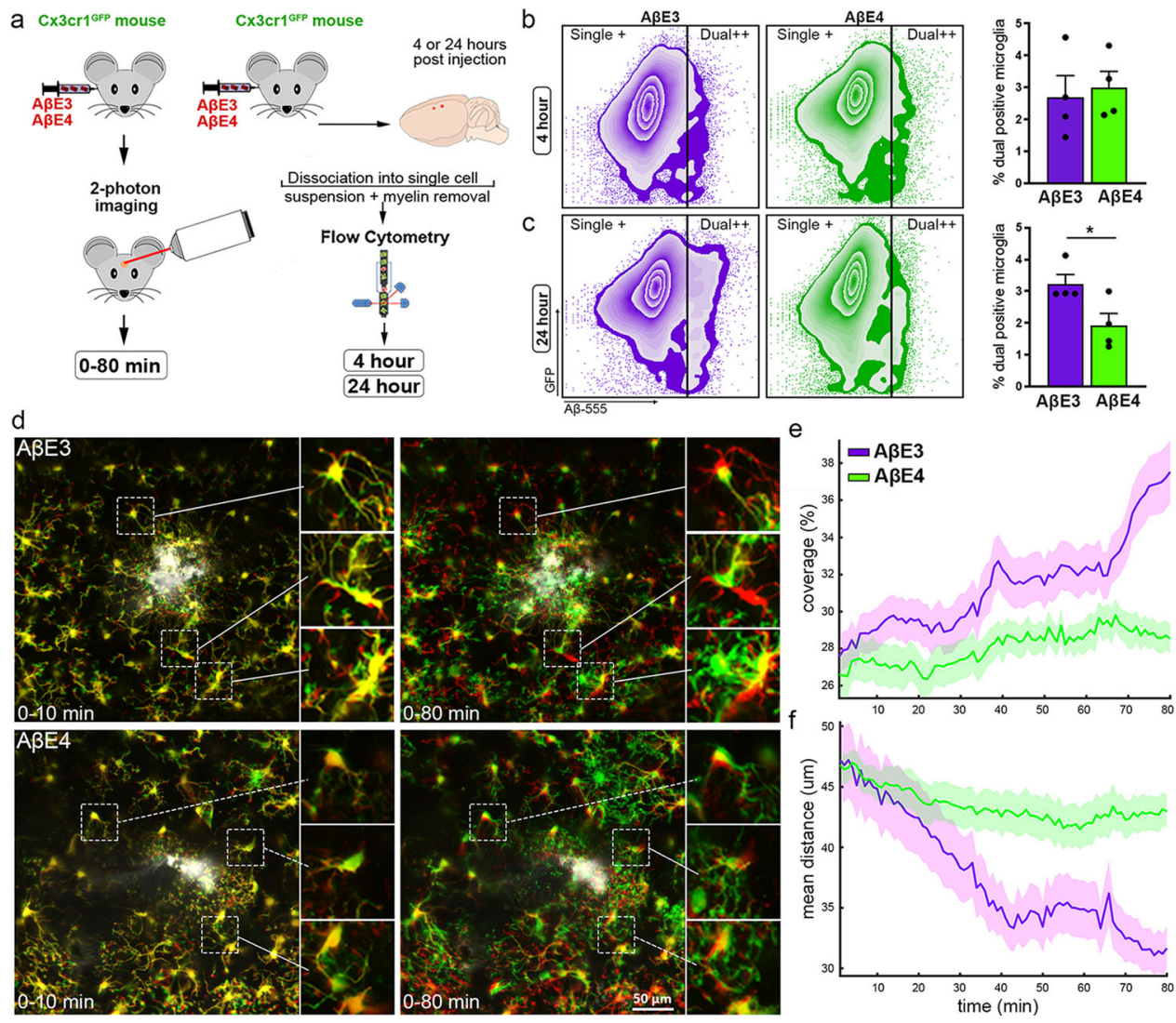

Fig. 3 Microglial interaction with $\mathbf{A} \boldsymbol{\beta}$ is affected by APOE isoform. $\mathrm{C} \times 3 \mathrm{cr} 1^{\mathrm{GFP}}$ mice were infused with Hi-Lyte Fluor 555-labeled A $(\mathrm{A} \beta-555$ )

preincubated with native E3 or E4. a Experimental design. b, c Four or $24 \mathrm{~h}$ post-injection the cortex surrounding the injection site was dissociated into a single cell suspension and used for flow cytometry $(\mathbf{b}, \mathbf{c})$ and FACS (experiment shown on Fig. 4). Flow cytometry zebra plots represent live microglia cells from injected mice that are GFPhigh/A $\beta-555^{\text {low }}$ and are counted as single + cells or GFPhigh/A $\beta-555^{\text {high }}$ cells counted as dual + cells. Flow cytometry zebra plots from $4 \mathrm{~h}(\mathbf{b})$ and $24 \mathrm{~h}(\mathbf{c})$ time points and bar plots correspond to the percentage of dual-positive microglia cells in A $\mathrm{EE} 3$ (purple) and A $\beta$ E4 (green) groups at 4 and 24 h. $p=0.0372$ by two-tailed unpaired $t$-test. $n=4$ mice/group. Bars represent mean \pm SEM. $\mathbf{d}$-f Two-photon imaging was used to track microglia movement in real time. $\mathbf{d}$ Representative fields of view from A $\beta E 3$ (top row) or A $\beta E 4$ (bottom row) infused mice. Time series are colorcoded with infused $A \beta$ as white; and initial time point in red and later time points in green as indicated on the panel, (0-10 or 0-80 min) with yellow representing the overlap between timepoints. Inserts show details of individual cells demonstrating the extent of migration and directionality of the cellular processes. Scale bar $=50 \mu \mathrm{m}$. e Percent coverage of infused $A \beta$ by microglial processes computed from channel overlap. $\mathbf{f} C$ hange of mean distance from microglia to infusion site over time for $A \beta E 3$ (purple) and A $\beta E 4$ (green) groups. The standard error within each group is plotted as a bounded line with mean as center line. $n=4$ mice/group.

in vivo two-photon imaging in $\mathrm{Cx} 3 \mathrm{cr} 1^{\mathrm{GFP}}$ mice injected with $\mathrm{A} \beta \mathrm{E} 3$ or $\mathrm{A} \beta \mathrm{E} 4$ (see Fig. 3a for the design). We injected the same volume and assessed the biodistribution post-injection by acquiring in vivo three-dimensional z-stacks (Supplementary Fig. 5d). For each animal, ZT-stack images were analyzed to quantify the microglial migration towards the infusion site and microglial coverage of the infusion site over time (Fig. $3 \mathrm{~d}-\mathrm{f}$ and Supplementary Fig. 5e, f). The representative images of the time series are color-coded with the initial timepoint $(0 \mathrm{~min})$ in red, later timepoints $(10$ or $80 \mathrm{~min}$ ) in green and the infused $A \beta$ in white (Fig. 3d and Supplementary Fig. 5e, f). The time series (Fig. 3d, insets) show clear separation of the green and red labels in the $A \beta E 3$ infusion group (Supplementary Movie 1), indicative of faster migration and better coverage of the infused $A \beta$ compared to A $\beta E 4$ (Supplementary Movie 2). In contrast, the time series from the mice infused with $\mathrm{A} \beta \mathrm{E} 4$ demonstrated a visible overlapping-yellow-colored-representing a slower migration velocity and less microglial $A \beta$ coverage. The percent coverage by the microglial processes over time was computed as the increase of the green channel intensity over the infusion site and shown on Fig. 3e. The change in the distance from microglia to the infusion site is presented on Fig. 3f. Microglia were able to extend processes towards the $\mathrm{A} \beta \mathrm{E} 3$ infusion over the first $40 \mathrm{~min}$. Once the processes reached the $A \beta E 3$, microglia formed lamellipodia that covered the $\mathrm{A} \beta \mathrm{E} 3$ throughout the remainder of the experiment ( $40-80 \mathrm{~min})$. In contrast, microglia demonstrated decreased capacity to extend processes towards the A $\beta E 4$ as well as encapsulate $\mathrm{A} \beta \mathrm{E} 4$ infusion. The results demonstrate that the infusion of $A \beta$ together with $E 3$ lipoproteins, unlike with $\mathrm{E} 4$, induces a more rapid response by microglia to isolate $\mathrm{A} \beta$ that suggests a protective mechanism diminishing the spread of $A \beta$.

E3 and E4 lipoproteins differentially affect microglia transcriptome in response to $\mathbf{A} \boldsymbol{\beta}$ infusion. Next, we performed RNA-seq on two FACS-sorted microglial populations (dual + and single + microglia) from $\mathrm{Cx} 3 \mathrm{cr} 1^{\mathrm{GFP}}$ mice injected with $\mathrm{A} \beta \mathrm{E} 3$ or $\mathrm{A} \beta \mathrm{E} 4$ (see Fig. $4 \mathrm{a}$ for the design). We assume that dual+ cells are in an active stage compared to their single + counterparts, which are either resting or in transition from homeostatic to active state. The goal was to examine how APOE isoform and the postinjection interval affects this transition. tSNE plots demonstrate that the expression profiles of single + and dual + microglia cluster into sharply defined groups for both timepoints (Fig. 4b). 

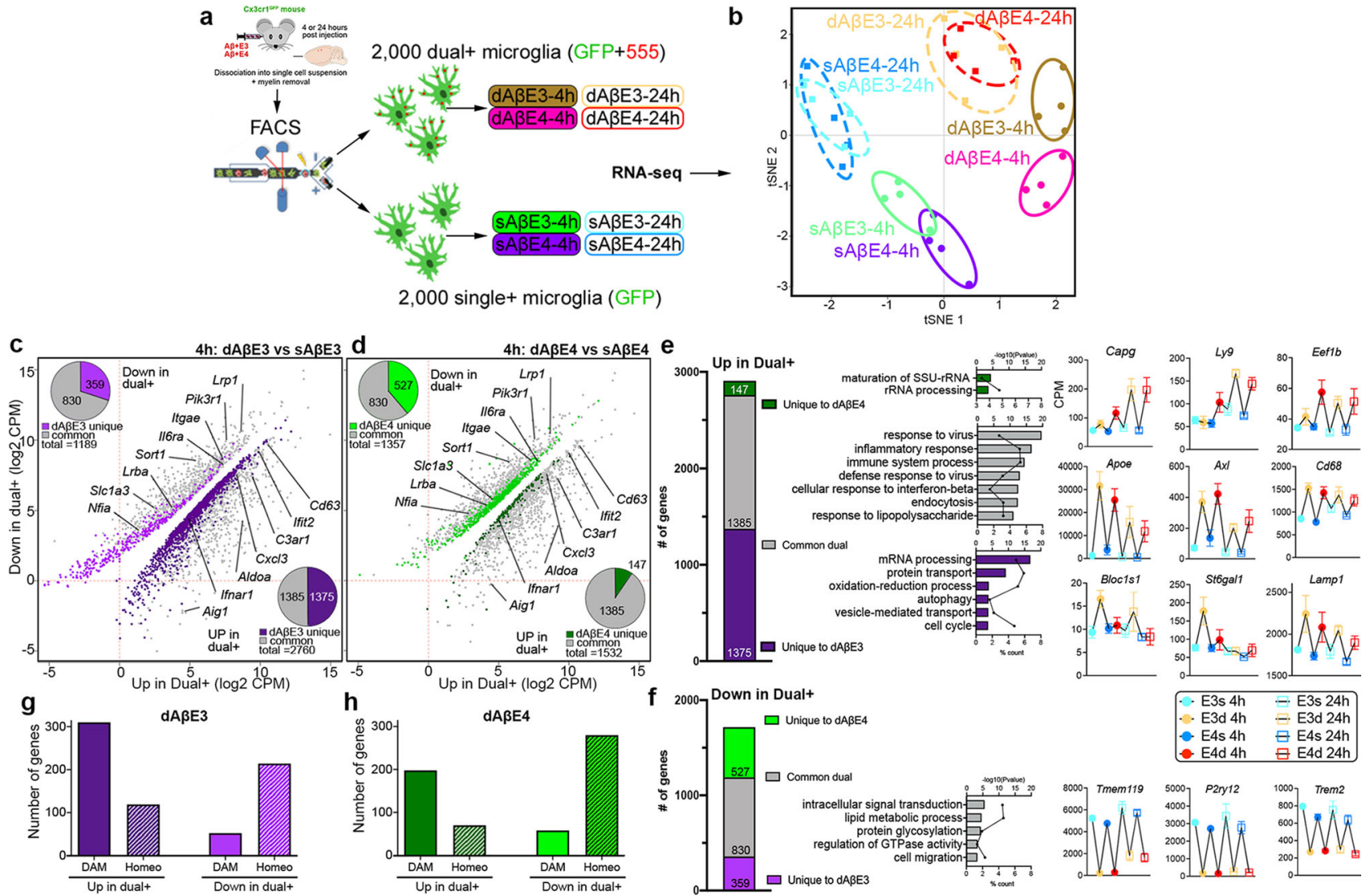

Fig. 4 A $\beta E 3$ and A $\beta E 4$ differentially affect microglia transcriptome. $C \times 3 c r 1 G F P$ mice were infused with $A \beta E 3$ or $A \beta E 4$, brain and processed followed by FAC sorting microglia at 4 and $24 \mathrm{~h}$ into single+ (referred to as sA $\beta E 3$ or sA $3 E 4$ ) and dual+ (dABE3 or dA $3 E 4$ ) populations and used for RNA-seq. a Experimental design. $\mathbf{b}$ tSNE plot shows clustering of dual + and single + microglia for four experimental groups. $n=4$ mice/group c, d Significantly affected transcripts for dual+ vs. single + microglia at $4 \mathrm{~h}$ time point (FDR $<0.05$ ) for $A \beta E 3$ (purple) and $A \beta E 4$ (green) groups. c DEGs in dA $\mathrm{EE3}$ vs. sA $3 \mathrm{E} 3$. $d D E G s$ in $d A \beta E 4$ vs. SA $\beta E 4$. Pi charts in the upper left (down in dual+), and lower right (up in dual+) represent genes common between, or unique to each APOE isoform. e, f Proportion of genes, GO terms and line patterning associated with common (gray) or unique genes between dA $\beta E 3$ vs. sA $\beta E 3$ (purple) and $\mathrm{dA} \beta \mathrm{E} 4$ vs. SA $\beta \mathrm{E} 4$ (green). e Common and unique upregulated in dual+ vs. single+. f Common and unique downregulated in dual + vs. single + . For both, bar plots indicate the $-\log 10 P$ value for each term, and the associated point in the center of each bar represents the percent of submitted genes found in each GO term. Line-patterning graphs show example of genes from each category. sA $\beta E 3$ light blue, dA $\beta E 3$ beige, sA $\beta E 4$ blue, dA $\beta E 4$ red; $4 \mathrm{~h}$ solid cirles, 24 h open circles. $\mathbf{g}, \mathbf{h}$ Bar graphs show the number of DAM (solid) and homeostatic (dot pattern) genes upregulated or downregulated in dual+ for $\mathrm{A} \beta \mathrm{E} 3$ ( $\mathbf{g}$, purple) or $\mathrm{A} \beta \mathrm{E} 4$ (h, green) injected mice.

Supplementary Fig. 6a, b demonstrates that numerous overlapping DEGs were commonly upregulated for the two timepoints in dual + vs. single + microglia. For each single + and dual+ populations, we identified genes with a similar level of expression at both timepoints (unchanged, gray), a group with higher expression at $4 \mathrm{~h}$ that declined in time (upregulated at $4 \mathrm{~h}$, blue), and a third group (red) with higher expression at $24 \mathrm{~h}$ (Supplementary Fig. 6c-f and Supplementary Data 2). Unchanged in dual+ cells were genes activated from the start with continuous expression and related to interferon signaling (Ifi204) or lipid transport $(L p l)$. Early-responding genes upregulated in dual + at 4 vs. $24 \mathrm{~h}$ were associated with inflammatory response, chemokine-mediated signaling, and chemotaxis (Supplementary Fig. 6c, d, blue color and Supplementary Fig. 6g). Genes that were upregulated at the later timepoint in dual+ microglia (lateresponding) were associated with protein folding, ribosome biogenesis, and glycolysis, consistent with the increased energy demands of processing the engulfed $A \beta$ (Supplementary Fig. 6c, d, red color and Supplementary Fig. 6g).

As seen from the tSNE plot on Fig. 4b, APOE isoform-specific effect on microglial transcriptomes was more pronounced at $4 \mathrm{~h}$. The examination of DEGs in dual + vs. single + microglia in both treatments revealed a higher number of total DEGs, specifically a higher number of upregulated genes in dual $+\mathrm{A} \beta \mathrm{E} 3$ microglia than in dual+ A $\beta E 4$ microglia (Fig. 4c, d, 2760, and 1532 respectively, pi charts dark purple and dark green). For both treatments, there were many shared/common DEGs upregulated in dual + vs. single + (Fig. 4e, f, gray boxes). In both $\mathrm{A} \beta \mathrm{E} 3$ and $\mathrm{A} \beta \mathrm{E} 4$ dual + microglia, there was an overall upregulation of DAM genes (186 genes) and downregulation of homeostatic genes (212 genes). Commonly upregulated in dual + microglia were DAM genes (Apoe and $A x l$ ) while classical homeostatic genes were downregulated (P2ry12 and Tmem119), indicating a profile characteristic for microglia in an active state (Fig. 4e, f). Also commonly upregulated in dual+ were receptors (Clec4 cluster, Marco, Axl, and Gas6), cytokines (nine genes), chemokines (12 genes), and genes related to phagocytosis and lysosomes (Cd68). Surprisingly, Trem 2 as well as several other DAM genes (Csf3r, Cebpa, and Ctss) had higher expression in single + microglia (respectively, downregulated in dual + ) in both treatments and at both timepoints. This suggests that Trem 2 is more associated with transition than the fully activated state. DAM genes uniquely upregulated for $\mathrm{A} \beta \mathrm{E} 3$ were genes associated with the lysosome/ autophagy process (Lamp1 and Bloc1s1), glycosylation (St6gal1), a glycosyltransferase implicated by GWAS with $\mathrm{AD}^{30}$, cytokines (Ccl6), and receptors (Clec4e and Clec12a). DAM genes uniquely 
a

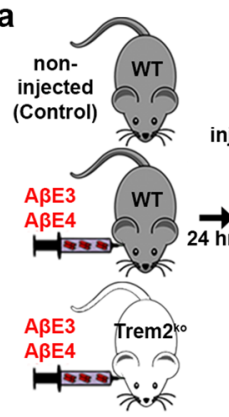

b

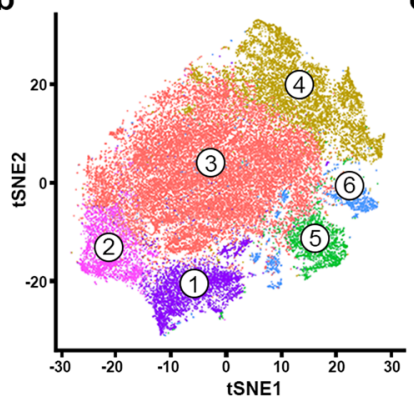

c

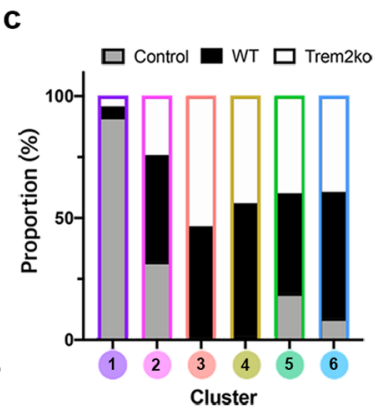

d

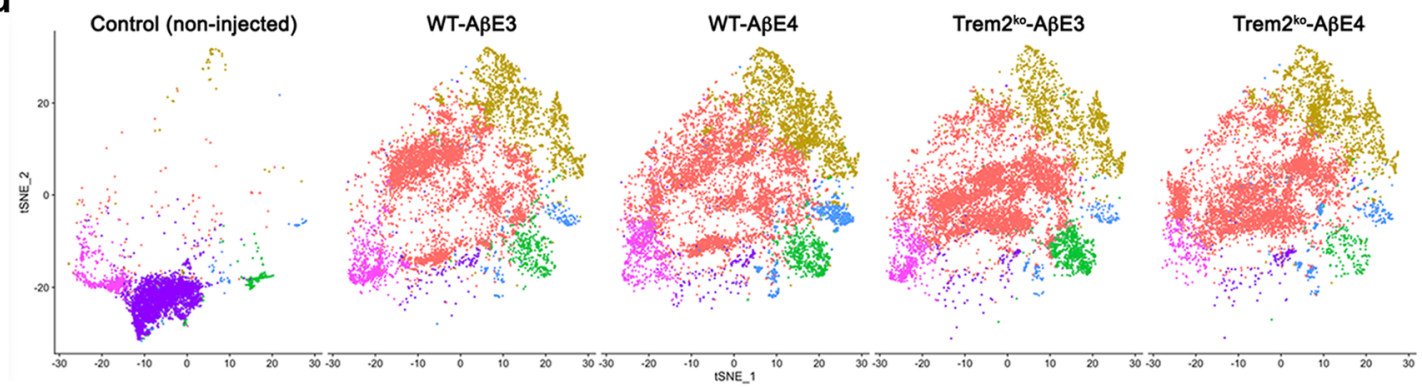

e

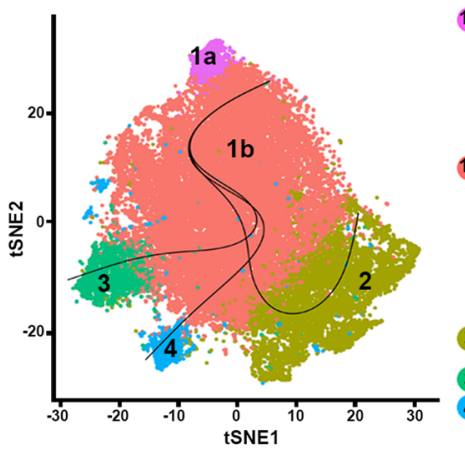

f

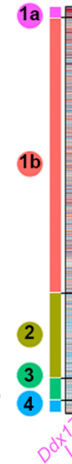

$\mathrm{CD}_{11 \mathrm{~b}^{+}}$

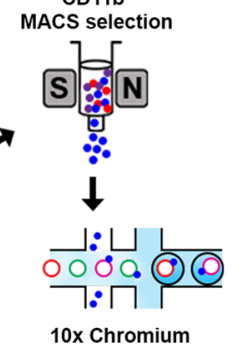

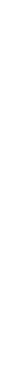
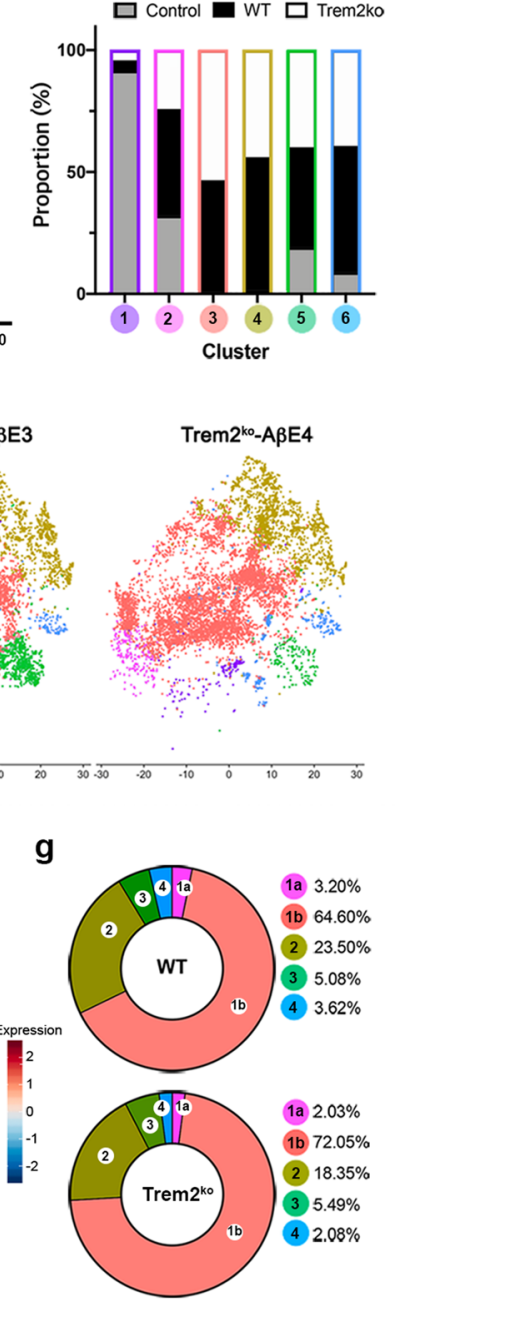

Fig. 5 scRNA-seq data reveal distinct microglial subpopulations in response to $\mathbf{A} \boldsymbol{\beta}$ infusion. a Schematic workflow of the experimental strategy for scRNA-seq. Microglia were isolated from WT and Trem $2^{\mathrm{ko}}$ mice injected with A $\beta E 3$ or A $\mathrm{EE} 4$ using CD11b immunomagnetic beads. Microglia from noninjected WT mice were used as control. Isolated cells were immediately applied to the 10x Chromium platform. $n=1$ for Control, $n=2$ for WT-A $\beta E 3$, WTA $\beta E 4$, Trem2 ${ }^{\mathrm{ko}}-\mathrm{A} \beta \mathrm{E} 3$, and Trem2 ${ }^{\mathrm{ko}}-\mathrm{A} \beta \mathrm{E} 4$. $\mathbf{b}$ t-SNE plot shows clusters of 39,898 microglial cells after re-clustering using Seurat. c Proportion of cells in each clusters from control, WT, and Trem2 ${ }^{\mathrm{ko}}$ microglia. $\mathbf{d}$ t-SNE plots of microglia from each experimental condition. e t-SNE plot shows clusters of 36,244 microglial cells after re-clustering of injected samples and inferred trajectories by Slingshot. $\mathbf{f}$ Heatmap shows the expression of top five genes in each cluster. $\mathbf{g}$ Proportion of cells in each cluster from WT and Trem2 ${ }^{\mathrm{ko}}$ microglia.

upregulated in $\mathrm{A} \beta \mathrm{E} 4$ were $\mathrm{Capg}$ (binds PIP2 and affects actin dynamic in macrophages ${ }^{31}$ ), Eef2 and Eef1b2 (regulate translation ${ }^{19}$ ) as well as Ly9 (lymphocyte antigen $9^{32}$ ). Overall, there was a higher number of DAM genes upregulated in dual+ $\mathrm{A} \beta \mathrm{E} 3$ (310) than in dual $+\mathrm{A} \beta \mathrm{E} 4$ (198) (Fig. 4g, h), suggesting that E3 could initiate a stronger response by microglia than E4.

Single cell sequencing identifies distinct microglial populations in response to $\mathbf{A} \boldsymbol{\beta}$ injection. To test how Trem 2 deficiency affects acute microglial response to $A \beta E 3$ or $A \beta E 4$ infused into the cortex of WT and Trem $2^{\text {ko }}$ mice, we performed scRNA-seq. Noninjected WT microglia were used as a control for the injection and analyzed in parallel (see Fig. 5a for the design). We collected a total of 60,046 sequenced cells with 2156 median genes expressed and 4988 median unique molecular identifiers (UMIs) per cell. Non-microglial cells were identified using cell typespecific genes and excluded from further analysis (Supplementary Fig. $7 \mathrm{a}-\mathrm{c}) ; 39,898$ microglial cells were re-clustered to catalog transcriptionally distinct subpopulations (Fig. 5b and Supplementary Data 3). We found that, excluding homeostatic clusters (clusters 1 and 2), four clusters consisted predominantly of microglia from $A \beta$ injected samples, suggesting these four clusters are affected by $\mathrm{A} \beta$ injection and not because of experimental artifacts, such as cell isolation (Fig. $5 c$, d and Supplementary Fig. 8). Thus, we re-clustered 36,244 microglial cells from injected groups, resulting in five clusters and then performed trajectory analysis to identify distinct activation states in response to the infusion (Fig. 5e and Supplementary Fig. 8d, e). We determined three potential trajectories (clusters 2, 3, and 4) from clusters 1a and $1 \mathrm{~b}$ (Fig. 5e). Sankey plot shown on Supplementary Fig. $8 \mathrm{f}$ shows cluster preservation between the two datasets.

To characterize heterogeneity within microglia, we identified cluster-specific genes that are differentially expressed between clusters (Fig. $5 \mathrm{f}$ and Supplementary Data 4). As seen in Fig. $5 \mathrm{f}$ and Supplementary Fig. 9c, the microglia in clusters 1a and $1 \mathrm{~b}$ showed increased expression of homeostatic signature genes, such as P2ry12, Tmem119, and Siglech ${ }^{19,33}$. However, cluster 1b showed 
an increase from the baseline (cluster 1a) expression of genes related to microglial activation such as Apoe, Cst7, Trem2, and Tyrobp and a slight decrease of homeostatic markers (Supplementary Fig. 9c). Thus, we characterized cluster $1 \mathrm{~b}$ as a transitional state. Interestingly, in WT mice Trem2 had the highest expression in cluster $1 \mathrm{~b}$, suggesting its involvement in the transition from homeostatic to active state. Genes associated with activated microglia were upregulated in clusters 2,3 , and 4 and accompanied by downregulation of homeostatic microglial genes. This suggests that clusters 2, 3, and 4 form in response to infusion of either $\mathrm{A} \beta \mathrm{E} 3$ or $\mathrm{A} \beta \mathrm{E} 4$ and present the active microglia state. Microglia in cluster 2 showed upregulation of genes related to chemotaxis (Ccl12, Ccl3, Ccl4, Cxcl16, Spp1, and Lgals3), inflammatory cytokine (Mif), lipoprotein lipase ( $L p l)$, and ribosomal components. Microglia in cluster 3 showed upregulation of pro-inflammatory genes, especially a set of genes important in NF- $\mathrm{BB}$ signaling such as Tnf, Illb, and Ptgs 2 as well as cytokines ( $\mathrm{Ccl} 3$ and $\mathrm{Ccl} 4)$. In cluster 4 , genes related to the interferon signaling pathway, including Ifitm 3, Ifi27l2a, Ifit1, and Irf7 as well as Apoe were highly expressed within immune system process as the top enriched GO term (Fig. 5f). We observed an increased proportion of cells in cluster $1 \mathrm{~b}$ and reduced proportion in cluster 2 in Trem2-deficient microglia compared to WT (Fig. 5g). These data demonstrate that in our acute model of brain injury, Trem 2 deficiency impairs the transition of microglia from homeostatic to an activated state, in the same way as chronic neurodegeneration ${ }^{17}$.

scRNA-seq identifies activated gene clusters downregulated in Trem $2^{\text {ko }}$ mice following $\mathbf{A} \boldsymbol{\beta}$ infusion. To identify differential transcripts, we compared microglia from Trem $2^{\mathrm{ko}}$ and WT mice and observed a higher number of DEGs in clusters 2, 3, and 4 associated with active microglia (Fig. 6a and Supplementary Data 5). Overall, there was reduced expression of immune response genes in Trem2-deficient microglia compared to WT microglia (Fig. 6b-d). In clusters 2, 3, and 4, DAM genes comprised $30-44 \%$ of the total number of DEGs that were upregulated in WT vs. Trem $2^{\text {ko }}$, and this was accompanied by the same decrease of homeostatic microglia genes (Fig. 6b-d), suggesting that microglia from Trem $2^{\mathrm{ko}}$ mice cannot adequately respond to the $A \beta E 3 / A \beta E 4$ infusion challenge. In cluster 2 the top downregulated genes in Trem $2^{\text {ko }}$ microglia were $L p l, S p p 1$, and $C d 9$; opposed by upregulation of canonical homeostatic genes such as P2ry12 (Fig. 6b). Clusters 3 (Fig. 6c) and cluster 4 (Fig. 6d) exhibited the largest number of downregulated genes in Trem $2^{\text {ko }}$ microglia and also showed the highest mismatch between WT and Trem $2^{\text {ko }}$ microglia in terms of homeostatic/DAM gene expression (see the bar graphs in Fig. 6c, d).

We validated the scRNA-seq data using fluorescent in situ hybridization (FISH) for Tmem119 and Adgre1 and spatially visualized their expression near the infusion site. We selected Tmem119 as a canonical microglia-specific gene that was not affected by Trem 2 deficiency, and Adgre1 (F4/80) was selected because it was significantly downregulated in Trem $2^{\text {ko }}$ microglia and was shown to be increased near the infusion site (see Fig. $2 \mathrm{k}-\mathrm{i}$ ). Similarly to the scRNA-seq data, we found a significant reduction in expression of Adgre 1 in Trem $2^{\mathrm{ko}}$ mice and no effect on Tmem119 level (Fig. 6e-g). Thus, FISH performed near the infusion site confirms the result for selected genes in the scRNA-seq datasets.

Trem2 deficiency and APOE isoform affects microglia response to $\mathbf{A} \boldsymbol{\beta}$. We next examined if the response to $\mathrm{A} \beta \mathrm{E} 3$ and $\mathrm{A} \beta \mathrm{E} 4$ infusion is APOE isoform- and/or TREM2-dependent. We compared microglia from WT and Trem $2^{\mathrm{ko}}$ mice injected with $\mathrm{A} \beta \mathrm{E} 3$ or $\mathrm{A} \beta \mathrm{E} 4$ within clusters 2,3 , and 4 which correspond to active microglia (Fig. 7a-c and Supplementary Data 6). For all clusters, we observed a higher number of homeostatic genes upregulated in Trem $2^{\mathrm{ko}}$ vs. WT microglia injected with A $\beta E 4$ compared to $A \beta E 3$-injected groups. Interestingly, the number of DEGs in Trem $2^{\text {ko }}$ vs. WT injected with $\mathrm{A} \beta E 4$ showed a larger difference compared to $\mathrm{A} \beta \mathrm{E} 3$-injected groups in clusters 2 and 4 . In cluster 2 , both $\mathrm{A} \beta \mathrm{E} 3$ - and $\mathrm{A} \beta \mathrm{E} 4$-injected Trem2 ${ }^{\mathrm{ko}}$ groups showed a decreased expression vs. their WT counterparts of genes such as Cxcl16, Lpl, Spp1, Marco, and Ly9, genes with roles in chemotaxis, endocytosis, and bioenergetic that could affect $A \beta$ uptake. Additional DEGs that were downregulated were several cytokines (Ccl3 and Ccl4), Fc receptors (Fcgr4), and Illb. In terms of genes upregulated in Trem $2^{\mathrm{ko}}$ vs. WT microglia in cluster 2 , A $\beta E 4$-injected Trem $2^{\text {ko }}$ showed a higher number of upregulated classic homeostatic genes such as P2ry12, P2ry13, and Tgfbl compared to A $\beta E 3$-injected Trem $2^{\mathrm{ko}}$ mice (Fig. $7 \mathrm{a}, \mathrm{A} \beta \mathrm{E} 4$ plot). Similarly, in cluster 4 there was a higher number of upregulated homeostatic genes in Trem $2^{\mathrm{ko}}$ vs. WT in A $\beta E 4$-injected compared to $\mathrm{A} \beta \mathrm{E} 3$-injected mice. For cluster 4 , in $\mathrm{A} \beta \mathrm{E} 4$-injected microglia we also observed a higher number of downregulated genes in Trem $2^{\mathrm{ko}}$ associated with interferon signaling (Ifit2, Ifi27l2a, Ifi207, Ifi204, and $A x l$ ) and endocytosis (Cd14, Cxcl16, Fth1, and Ifitm3). In contrast, in cluster 4, there were very few DEGs in A $\beta E 3$-injected Trem $2^{\mathrm{ko}}$ vs. WT mice. These data suggest a possible interaction between TREM2 and APOE-isoform that could affect microglial chemotaxis and interferon signaling pathway.

To test how Trem 2 deficiency and APOE native particles affect A $\beta$ uptake, we used primary microglia from WT and Trem $2^{\mathrm{ko}}$ mice. As expected, there was a significant difference in uptake between WT-A $\beta E 3$ and WT-A $\beta E 4$. Surprisingly, we found that Trem 2 deficiency significantly decreased $A \beta$ uptake only in $A \beta E 4-$ but not in A $\beta E 3$-treated cells (Fig. 7d, e). Following this, we used primary microglia isolated from mice expressing human E3 and E4 and compared to their Trem $2^{\mathrm{ko}}$ counterparts (E3/Trem $2^{\mathrm{ko}}$ or $\left.\mathrm{E} 4 / \mathrm{Trem} 2^{\mathrm{ko}}\right)$. Cell were treated with $\mathrm{A} \beta$ alone without the addition of E3 and E4 native particle. As seen on Supplementary Fig. 10, both APOE4 isoform and Trem2 deletion significantly decreased $A \beta$ uptake, and the overall reduction of $A \beta$ uptake by E4/Trem $2^{\text {ko }}$ microglia was reduced fivefold compared to E3expressing microglia. We speculate that the addition of native E3/ E4 lipoproteins serves as a ligand to TREM2 or other microglial receptors and facilitates $A \beta$ uptake which is restored to normal with native E3 lipoproteins in Trem $2^{\mathrm{ko}}$. Overall, we conclude that Trem 2 deficiency may further exaggerate E4 lipoprotein insufficiency to activate microglia and phagocytize $A \beta$.

\section{Discussion}

In this study, we identified a common phospholipid signature between major phospholipid classes of native E3 and E4 lipoproteins and brain samples from $A P O E \varepsilon 3 / 3$ and $A P O E \varepsilon 4 / 4 \mathrm{AD}$ patients. Our results support the hypothesis that native E3 lipoproteins trigger a more effective microglial response to $A \beta$ than E4 in ameliorating $A \beta$ deleterious effect. We posit that the divergence in phospholipid content of E3 and E4 native lipoproteins affects $A \beta$ binding to microglia receptors in an isoformspecific manner and subsequently has a differential impact on microglia transcriptome (Fig. 8). Consequently, E3 induces a faster microglial response than $\mathrm{E} 4$ characterized by rapid process extension allowing $A \beta$ to be isolated and engulfed. Lastly, behavioral testing following $A \beta$ injections demonstrated that $E 3$ native lipoproteins exhibited a better protection than E4 in diminishing $\mathrm{A} \beta$ effects on memory.

The quantitative assessment of the phospholipid composition of E3 and E4 native lipoproteins and brain samples from 

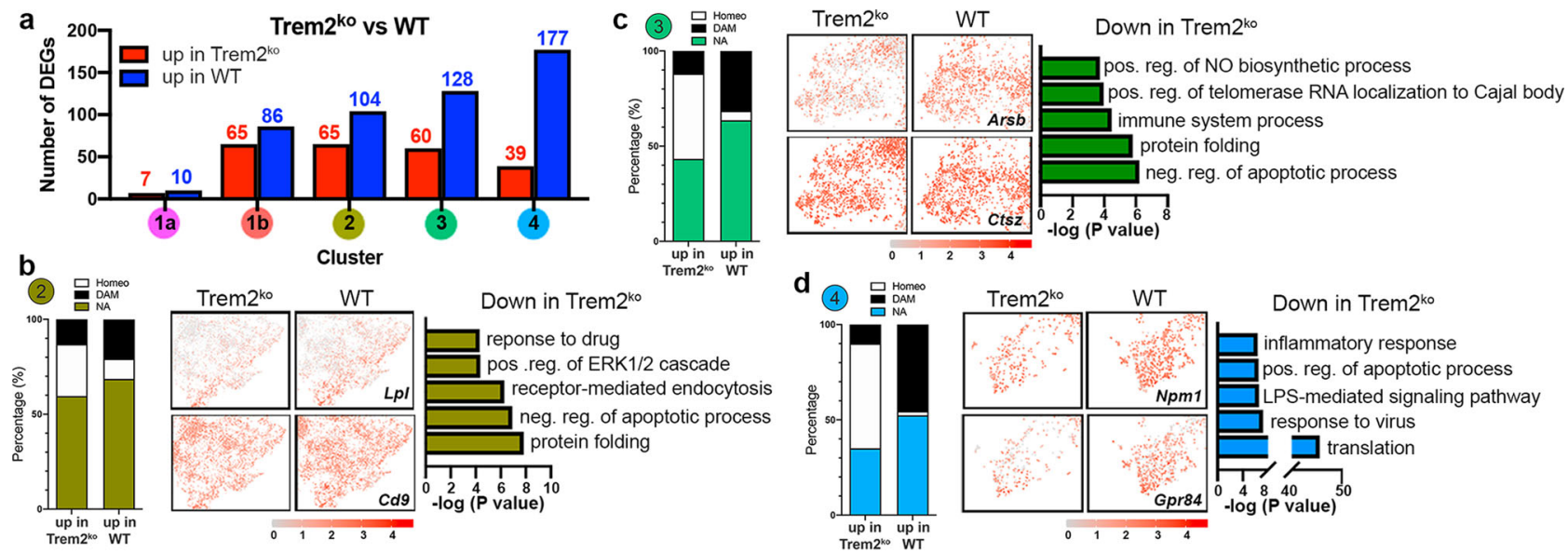

Down in Trem2 $2^{\mathrm{ko}}$
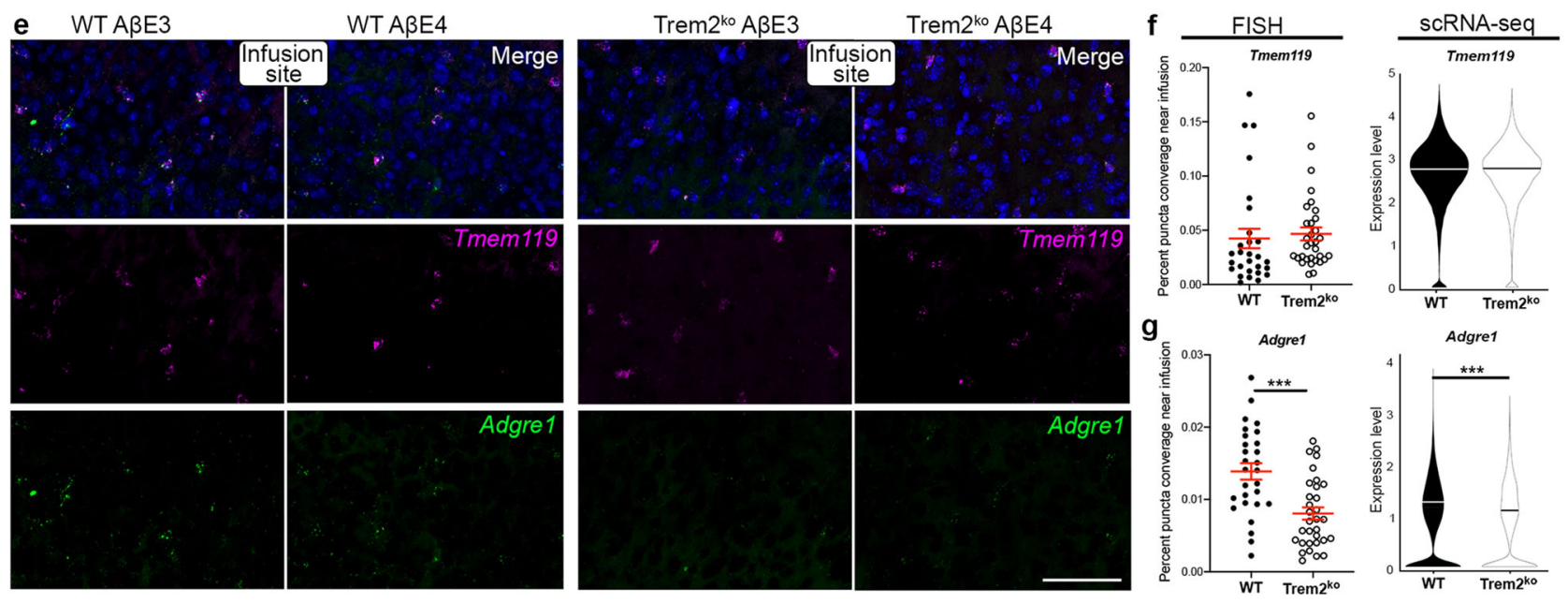

Fig. 6 scRNA-seq identifies activated gene clusters downregulated in Trem2 ${ }^{\mathbf{k} 0}$ mice following A $\beta E 3$ or A $\beta E 4$ infusions. a The number of DEGs in Trem2 ${ }^{k o}$ compared to WT in each cluster (Bonferroni-corrected $p$ value $<0.05$ and natural $\log F C>0.1$ ). b-d The proportion of homeostatic (Homeo) or DAM genes among DEGs in Trem2 ${ }^{\mathrm{ko}}$ or WT groups in clusters 2 (b), 3 (c), and 4 (d). Feature plots (Left) showing representative genes and bar plot (Right) showing top five GO terms that are significantly downregulated in Trem2 ${ }^{\mathrm{ko}}$ compared to WT microglia in the three infusion responsive clusters. $n=4$ per group (WT and Trem2 ${ }^{\mathrm{ko}}$ ). $\mathbf{e}-\mathbf{g}$ The expression of Tmem119 and Adgre1 was spatially visualized at $200 \mu \mathrm{m}$ perimeter from the injection site. e Representative images of FISH analyses of microglial gene expression near the A $\beta$ infusion site. (Tmem119 - pink, Adgre1 - Green, Nuclei - Blue). Scale bar $=50 \mu \mathrm{m} . \mathbf{f}, \mathbf{g}$ Scatter dot plots showing the area of puncta coverage adjacent to the infusion site along with violin plots of the expression of the same gene in the scRNA-seq dataset for Tmem119 (f), and $\operatorname{Adgre1}(\mathbf{g}) . n=8$ mice per group (equal males and females), with four infusion sites analyzed per animal, red lines represent mean \pm SEM, ${ }^{\star \star \star} p<0.001(p=0.0001)$ using two-tailed unpaired $t$-test.

$A P O E \varepsilon 3 / 3, \quad A P O E \varepsilon 4$ carriers, and APOE\&4/4 AD patients demonstrated a significant APOE isoform-specific difference in phospholipid profiles. Interestingly, our analysis demonstrates a very strong correlation of the phospholipid profile of human and native lipoprotein samples (Fig. 1c). It should be noted that the human brain samples include lipids that are structural part of cellular membranes as well as lipoproteins in interstitial fluid. Regardless that the source of the phospholipids was not entirely the same in human brain parenchyma and native lipoproteins isolated from ACM, it was remarkable to discover such a high level of similarity in the direction of fold change between the phospholipids of human samples and native lipoproteins that is APOE isoform-specific. As shown on Fig. 1a, several of the phospholipid classes (PE, PI, PS, SM, and PA) demonstrate an apparent $A P O E \varepsilon 4$ allele dose-response effect in the order $A P O E \varepsilon 3 / 3>\varepsilon E 3 / 4>\varepsilon E 4 / 4$.

As the major lipid carrier in the brain APOE lipoproteins play an important role in the chaperoning of cholesterol and phospholipids between cells and serving as a ligand for multiple immune receptors such as TREM2. It has been shown that TREM2 signaling is activated by phospholipids such as PI and
$\mathrm{PC}^{34}$ and TREM2 binds APOE as well as LDL and $\mathrm{HDL}^{22}$. Our lipidomic results demonstrate that four major phospholipid classes including neutral (PI and PC) and negatively charged (PE and PS) are significantly reduced in E4 lipoproteins when compared to E3, but not cardiolipin. Sensing of lipids is important for microglia activation and affects functions such as chemotaxis and phagocytosis (reviewed in Chausse et $\mathrm{al}^{35}$.). Increasing evidence suggests that brain phospholipid levels decrease with aging as well as during $\mathrm{AD}$ progression ${ }^{36}$, making the initial deficit we have identified in E4 particles even more detrimental with the progression of the pathology. The reduced lipidation of $\mathrm{E} 4$ compared to $\mathrm{E} 3$ native particles may impact $A \beta$ aggregation which in turn could affect cognitive performance and microglia activation state $^{37}$. Numerous studies demonstrated APOE binds to $A \beta$ with high affinity and most agree that there is an isoform-specific effect on $\mathrm{A} \beta$ aggregation $\mathrm{E} 4>\mathrm{E} 3>\mathrm{E} 2^{38,39}$. Furthermore, studies have suggested that $\mathrm{E} 3$ forms more stable complex with $\mathrm{A} \beta$ than $\mathrm{E} 4^{39}$ however in the present study we did not examine the stability of A $\beta$-APOE complex. Our data demonstrated that both E3 and $\mathrm{E} 4$ native lipoproteins precluded the formation of $\mathrm{A} \beta$ oligomers (Supplementary Fig. 2a, b). However, we cannot exclude a 

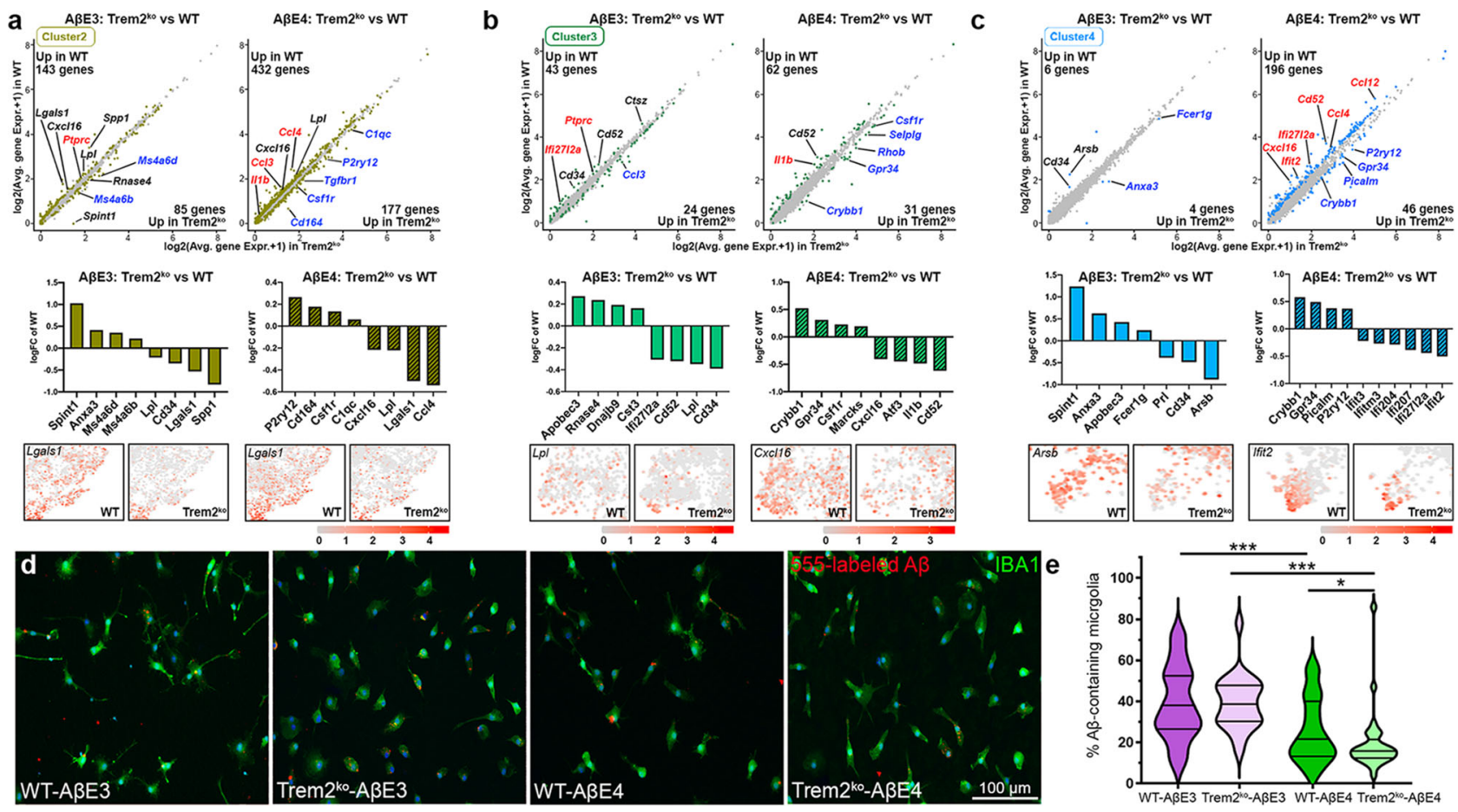

Fig. 7 Trem2 deficiency and APOE isoform affect microglia response to Aß. a-c Scatter plots, bar plots, and feature plots showing the differentially expressed genes in microglia from WT and Trem $2^{\mathrm{ko}}$ mice injected with A $\beta$ E4 or A $\beta$ E3 complexes for cluster 2 (a), cluster 3 (b), and cluster 4 (c). Differentially expressend genes at $p<0.01$ are colored in each cluster color. The adjusted $P$ value was performed using Bonferroni correction. Genes labeled with black color indicate commonly up- or down-regulated genes in A $\beta E 3$ or A $\beta E 4$ injected mice. Genes labeled with red indicate uniquely upregulated genes in WT-A $\mathrm{EE} 3$ or WT-A $\beta E 4$ and genes labeled blue is uniquely upregulated genes in Trem $2^{\mathrm{ko}}-\mathrm{A} \beta \mathrm{E} 3$ or Trem $2^{\mathrm{ko}}-\mathrm{A} \beta \mathrm{E} 4$. $n=2$ mice per group (WTA $\beta E 3$, WT-A $\beta E 4$, Trem2 ${ }^{\mathrm{ko}}-\mathrm{A} \beta E 3$, and Trem2 $\left.2^{\mathrm{ko}}-\mathrm{A} \beta \mathrm{E} 4\right)$ ). d, e Microglia established from WT and Trem2 ${ }^{\mathrm{ko}}$ mice were treated with and 555-labeled $A \beta$ for $1 \mathrm{~h}$. d Representative images of microglia from WT and Trem $2^{\mathrm{ko}}$ mice incubated with $A \beta E 4$ or A $\beta E 3$ labeled with IBA1 in green and 555-labeled A $\beta$ in red. e Bar plot showing the average percentage of $A \beta$-containing microglia for each group (WT-A $\beta E 3$ purple, WT-A $\beta E 4$, green, Trem2 ${ }^{\mathrm{ko}}-\mathrm{A} \beta E 3$ light purple, and Trem2 ${ }^{\mathrm{ko}}$-A $\beta E 4$ light green). $n=3$ independent cultures at least in triplicate. Analysis by one-way ANOVA followed by Tukey's multiple comparison test. WT-A $\beta$ E3 vs. WT-A $\beta$ E4, $p=0.0008$; Trem2 ${ }^{\mathrm{ko}}-\mathrm{A} \beta \mathrm{E} 3$ vs. Trem2 ${ }^{\mathrm{ko}}-\mathrm{A} \beta \mathrm{E} 4, p<0.0001$; WT-A $\beta$ E4 vs. Trem2 ${ }^{\mathrm{ko}}$-A $\beta$ E4, $p=0.0485$. Violin plots represent kernel densities for each dataset showing median (middle line) and 75\% (top) and 25\% percentile (bottom). ${ }^{\star} p<0.05$; ${ }^{\star \star \star} p<0.001$.

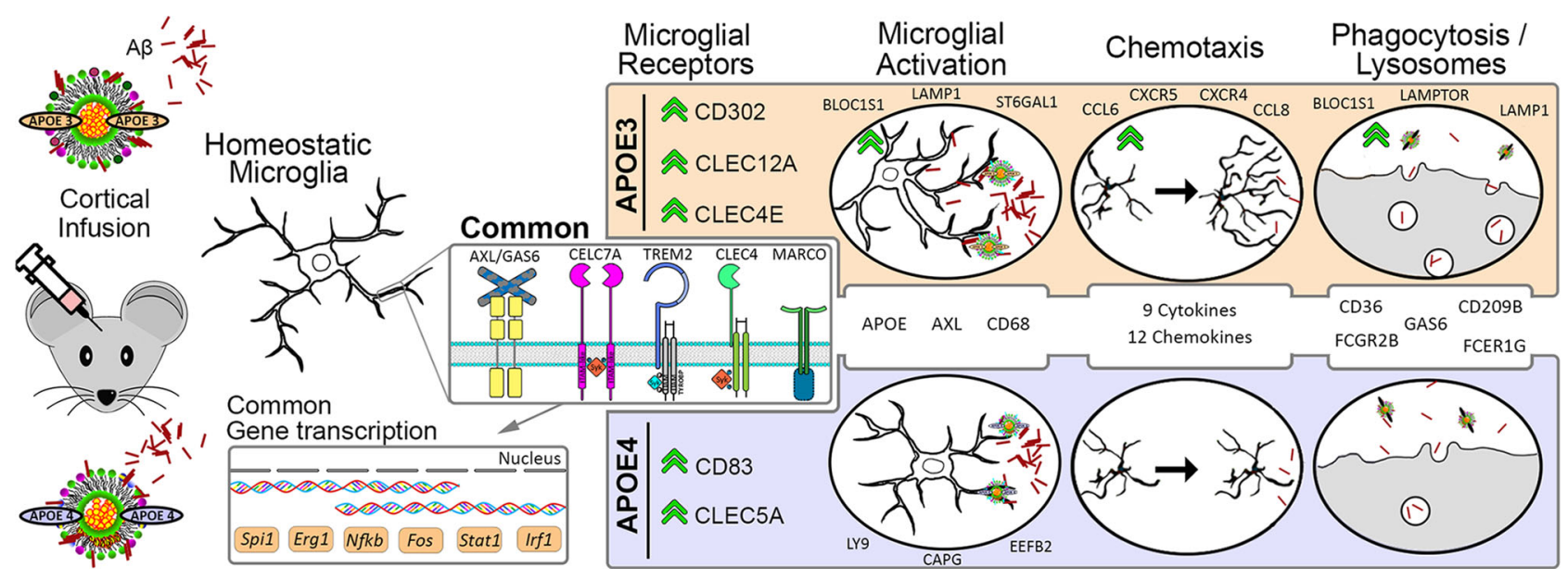

Fig. 8 Illustration of the APOE isoform-specific phospholipid composition effects on microglia transcriptome and phenotype in response to A $\beta$. Shotgun and LC-MS lipidomic analysis of APOE3 and APOE4 astrocyte conditioned media identified isoform-specific differences in phospholipid composition of APOE-containing lipoproteins. E3 or E4 lipoproteins co-infused with A $\beta$ differentially impacted A $\beta$ binding to microglia receptors ( $A X L$, different CLECs clusters, TREM2, CD83, and others) this in turn affects genes expression of transcription factors (Spi1, Stat1, Nfkb, and Irf1) resulting in microglia activation. Active microglia demonstrate characteristic transcriptional profile, increased secretion of cytokines and chemokines thus facilitating chemotaxis and phagocytosis. Green arrows indicate an upregulation of that gene or category compared to the other APOE isoform. 
possible presence of occasional $A \beta$ oligomers and potentially an isoform-specific effect on $\mathrm{A} \beta$ oligomerization that could be detected using quantitative methods with higher resolution. In confirmation of such possibility is the fact that E3 was more effective than $\mathrm{E} 4$ in rescuing cognitive deficits caused by $\mathrm{A} \beta$ oligomers (Fig. 2a-d). In terms of effects on behavior, we propose that microglia in contact with APOE3 native lipoproteins are more protective by restricting or eliminating the harmful effects of $\mathrm{A} \beta$ oligomers on the neural cells and circuits involved in cognitive function.

In terms of APOE effect on microglia, we found that E3 native lipoproteins are more effective than $\mathrm{E} 4$ in triggering microglia response to $A \beta$ injections. The transcriptomic profile of microglia isolated from $\mathrm{A} \beta \mathrm{E} 3$ injected mice have a higher enrichment of gene expression networks associated with innate immune response compared to microglia from $\mathrm{A} \beta \mathrm{E} 4$ injected. To address the transcriptional changes in phagocytic and non-phagocytic microglia we compared dual+ vs. single + at 4 - and 24 -h postinjection. Transcriptionally, the dual+ populations are very different from single + at both 4 - and 24-h post-injection, representing an activated state with upregulation of DAM genes and downregulation of homeostatic genes. We further subdivided dual + population into early-responding genes ( $4 \mathrm{~h}$; Ccl9, Ifit1, and $C t s b$ ), genes with continuous expression (Ifi204, Lpl, and Il10), or late-responding genes (24 h; Adgre1, Mif, and Ccl2). By separating of microglia on dual + and single + we conclude that the APOE- isoform-specific effects are more pronounced at earlier points of the injury that may reflect involvement in the initial recognition of $A \beta+A P O E$ complexes by microglia. Furthermore, in $\mathrm{Cx} 3 \mathrm{cr} 1$ mice (Fig. 4), A $\beta \mathrm{E} 3$ injections were associated with more active transcriptomic profile comprised by differential increase of DAM compared to A $\beta E 4$ injections. Recently, Ngueyn et $\mathrm{al}^{40}$. using single nuclei RNA-seq of human AD brain identified microglia subpopulation annotated as "amyloid-responsive microglia" (ARM), which was enriched in APOE\&3/3 patients and relatively depleted in $A P O E \varepsilon 4$ carriers. Ngueyen et al. propose $\mathrm{ARM}$ as primed to elicit an activated immune response and note the reduced ARM response in $A P O E \varepsilon 4$ carriers $^{40}$. We found a number of overlapping genes between our dual + cells (Fig. 4) and injury responsive microglia from Hammond et $\mathrm{al}^{33}$., which contain genes associated with interferon signaling. We found that the number of overlapped genes is higher in dual+ microglia from $A \beta E 3$ at $4 \mathrm{~h}$ than in dual $+\mathrm{A} \beta \mathrm{E} 4$, suggesting $\mathrm{E} 3$ induces the change of the microglial phenotype from homeostatic state to interferon responsive microglia better than $\mathrm{E} 4$. We also compared our common dual+ signature (Fig. 4) to the list of genes identified by Krassmann et $\mathrm{al}^{41}$. as associated to microglia phagocytic function. We identified a similar pattern of overlapping genes such as increased expression of Apoe, $A x l$, and decreased expression of P2ry12 and Tmem119 that were commonly affected in $\mathrm{A} \beta \mathrm{E} 3$ and $\mathrm{A} \beta \mathrm{E} 4$ injected mice. We speculate that there is a commonality between the effect of E3 and E4 native lipoproteins on phagocytic transcriptomic profile.

The identified transcriptional changes are accompanied by phenotypic changes in microglial morphology exhibited by increased process orientation towards the infusion site in $\mathrm{A} \beta \mathrm{E} 3$ vs. $\mathrm{A} \beta \mathrm{E} 4$ injected mice and an increase in microglia which colocalized with $A \beta$. We confirmed this using in vivo two-photon imaging, demonstrating that within the first hour of infusion microglia exposed to $A \beta E 3$ extended processes toward $A \beta$ faster than microglia exposed $A \beta E 4$. Thus, in brains injected with $A \beta E 3$, microglia can cover and envelop $A \beta$ with better efficiency than microglia from $A \beta E 4$-injected brains (Fig. 3). Finally, in vivo and in vitro experiments show that E3-native particles are more effective than E4 at promoting $\mathrm{A} \beta$ uptake (Figs. 3, 7 and Supplementary Fig. 4). Furthermore, as shown on Supplementary
Fig. 3, APP mice expressing human APOE3 have a higher microglia coverage compared to their APOE4 expressing counterparts and this is accompanied by different morphology. We posit that differences in microenvironment around amyloid plaques, e.g., the composition of APOE-containing lipoproteins, may affect microglia response to amyloid aggregation in plaque form. Our interpretation of the findings from acute $A \beta$ injections and chronic amyloid deposition is that E3 better mobilizes microglia to form a protective barrier surrounding the neuronal damage caused by the $A \beta$ injection and restricts its spread by increasing A $\beta$ uptake.

Recently, we reported that microglia-plaque coverage of small plaques is more efficient at restricting plaque-growth than of larger plaques ${ }^{24}$, and this effect is modulated by both APOE isoform as well as TREM2 status. This emphasizes the importance of the early response by microglia to amyloid and implicates the phenotypic response seen in the first $24 \mathrm{~h}$ in the A $\beta E 3$ mice as critical to the long-term protection from amyloid buildup and eventual neuronal damage. Studies addressing the microglia phenotypic response in TREM2-deficient mice have shown impaired ligand binding, reduced cell clustering to amyloid, reduced plaque compaction, and an impaired activation profile ${ }^{19,21,24,34,42}$. Using scRNA-seq we identify clusters 2,3 and 4 as infusion-responsive clusters characterized by downregulation of homeostatic microglial genes and upregulation of DAM genes as well as other genes associated with active microglia phenotype ${ }^{19,33,41,43}$ (Fig. 6). Interestingly, Trem2-deficient microglia showed impairment of the transitioning toward the activation state (cluster $1 \mathrm{~b}$ on Fig. 5) and overall higher expression of homeostatic microglial genes in infusion-responsive clusters (Fig. 6), indicating a defective response to acute $A \beta$ injection. Finally, across all the activated clusters we find larger differences of gene expression in $\mathrm{A} \beta \mathrm{E} 4$-injected mice depending on the presence of TREM 2 compared to $A \beta E 3$-injected groups. In clusters 2 and 4, whereas both A $\beta E 3$ - and A $\beta E 4$-injected Trem $2^{\mathrm{ko}}$ groups showed a decrease in expression of genes associated with activation vs. their WT counterparts, A $\beta E 4$-injected Trem $2^{\mathrm{ko}}$ microglia showed a higher number of upregulated classic homeostatic genes compared to $\mathrm{A} \beta \mathrm{E} 3$-injected Trem $2^{\mathrm{ko}}$ mice. Interestingly, the number of DEGs in Trem $2^{\mathrm{ko}}$ vs. WT injected with $A \beta E 4$ showed a larger difference compared to $A \beta E 3$-injected groups in cluster 4 . For this cluster, $A \beta E 4$-injected microglia showed a higher number of downregulated genes in Trem $2^{\text {ko }}$ microglia, primarily genes associated with interferon signaling. In vitro uptake experiments, also surprisingly, demonstrated that Trem 2 deficiency significantly decreased $A \beta$ uptake only in $A \beta E 4-$ but not in $A \beta E 3$-treated microglia. These data suggest a possible interaction between TREM2 and APOE-isoform that could affect microglial chemotaxis and interferon signaling pathways.

Collectively, we found that in the presence of $A \beta, E 3$ lipoproteins are more effective than E4 lipoproteins at inducing rapid transcriptional and phenotypic response by microglia, that can counteract $\mathrm{A} \beta$ harmful effects.

\section{Methods}

Experimental models. All animal experiments were performed in accordance with the NIH Guide for Care and Use of Animals and approved by the University of Pittsburgh Institutional Animal Care and Use Committee. The following mouse lines were bred to generated experimental mice: Wild-type C57BL/6 J (WT; JAX), human APOE3 and APOE4 targeted replacement mice (E3 and E4; Taconic),

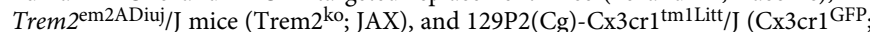
JAX). Human APOE3 and APOE4 targeted replacement mice were bred to

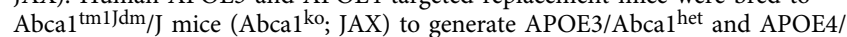
Abcal ${ }^{\text {het }}$ (E3 ${ }^{\text {het }}$ and E4 ${ }^{\text {het }}$ ). Likewise, human APOE3 and APOE4 targeted repla-

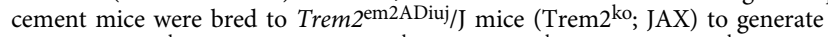
APOE3/Trem $2^{\text {ko }}$ and APOE4/Trem $2^{\text {ko }}$ (E3/Trem $2^{\text {ko }}$ and E4/Trem $2^{\text {ko }}$ ). Furthermore, APP/PS1dE9 mice expressing mutant familial variants of human amyloid precursor protein (APP; JAX) with Swedish mutation, and human presenilin 1 
carrying the exon-9-deleted variant (PSEN1dE9) were crossed to human APOE3 and APOE4 targeted replacement mice. Genotypes were confirmed with primers on Supplementary Table 2. All experimental mice were on the C57BL/6 genetic background, kept on a $12 \mathrm{~h}$ light-dark cycle with ad libitum access to food and water and randomly assigned to an experimental group. All reagents were purchased from Fisher Scientific unless documented otherwise. Methoxy-X34 (X34) 1,4-bis (3-carboxy-4-hydroxyphenylethenyl)-benzene, was provided by William $\mathrm{E}$ Klunk, MD, $\mathrm{PhD}$, (University of Pittsburgh). For in vivo labeling of dense plaques, we used Methoxy-X04 (X04) 1,4-bis (4'-hydroxystyryl)-2-methoxybenzene, synthesized in the Klunk lab and used according to our previous publication ${ }^{24}$.

AD brain samples. Human samples (Supplementary Table 1) were provided by the University of Pittsburgh Alzheimer's Disease Research Center (ADRC) brain bank and the Sanders-Brown Center on Aging at the University of Kentucky. The samples were collected according to the protocols approved by the University of Pittsburgh Institutional Review Board and Committee for Oversight of Research Involving the Dead (CORID, No 731). Braak staging was performed on Bielschowsky-stained sections and $A P O E$ allelic polymorphism previously determined by a PCR-based assay. Gray matter samples of $A P O E \varepsilon 3 / 3, A P O E \varepsilon 3 / 4$, and $A P O E \varepsilon 4 / 4$ genotypes from the right inferior parietal lobule were dissected and used for MDMS-SL. Age and postmortem intervals (PMI) matching was confirmed by $t$ test.

Native APOE generation and characterization. Cultures of primary astrocytes were established from 1-day-old E3, E4, E3 ${ }^{\text {het }}$, and E4 $4^{\text {het }}$ targeted replacement pups $^{44,45}$. The cortices and hippocampi were mechanically dissociated using a sterile pasteur pipette and cultivated in DMEM/F12 medium supplemented with $10 \%$ bovine growth serum, L-glutamine and antibiotics. Cells were cultured on poly-D-lysine $(100 \mu \mathrm{g} / \mathrm{mL})$ coated T75 Costar flasks (Corning). Confluent primary astrocytes were incubated with de-lipidated serum treatment medium (Neurobasal medium with antibiotics and glutamine as above minus serum) for $48 \mathrm{~h}$. Conditioned medium were collected immediately centrifuged, washed, and filtered through $0.22 \mu \mathrm{m}$ filter and concentrated using Amicon ${ }^{\circledast}$ Ultra Centrifugal Filters (10 $\mathrm{kD}$ cutoff) to retain relatively large particles (between $8-12 \mathrm{~nm}$ diameter). Native APOE lipoproteins were washed three times with cold PBS using Amicon ${ }^{\circledR}$ Ultra Centrifugal Filters and their concentration measured by quantitative Western Blotting (NUPAGE) using commercial human APOE (Meridian Life Science) as a standard. Native APOE particles were resolved on Novex ${ }^{\mathrm{m}} 4-20 \%$ Tris-Glycine gels using an Amersham ${ }^{\mathrm{m}}$ HMW calibration kit as native ladder (GE Healthcare). The size of APOE lipoprotein particles were measured by dynamic light scattering with Zetasizer Nano Z (Malvern). APOE lipoprotein total cholesterol, free cholesterol, and cholesterol ester content was assessed with the Cholesterol Quantitation Kit (Sigma) according to the manufacturer's instructions. Total phospholipid content of mouse CSF was assessed with the Phospholipid Assay Kit (Sigma) according to the manufacturer's instructions.

Multi-dimensional mass spectrometry shotgun lipidomics (MDMS-SL). The MDMS-SL assay ${ }^{46-48}$ was performed to determine differences in the lipid composition of the native $\mathrm{E} 3$ and $\mathrm{E} 4$ particles isolated from primary astrocyte cultures and human $\mathrm{AD}$ brain samples described above. APOE particles isolated from $\mathrm{E} 3^{\text {het }}$ and $\mathrm{E} 4^{\text {het }}$ primary astrocytes, exhibiting diminished APOE lipidation states, were used as negative controls. Quantitative analysis was performed on a triplequadrupole mass spectrometer (Thermo Fisher Scientific) equipped with an automated nanospray apparatus NanoMate and Xcalibur system. Internal standards for the quantification of individual molecular species of the major lipid classes were added to each sample at the start of the extraction procedure. Lipid extraction was performed by the methyl-tert-butyl ether (MTBE) method with resuspension in chloroform/methanol $(1: 1 \mathrm{v} / \mathrm{v})$ solution with nitrogen flush. Identification and quantification of all reported lipid molecular species scans from the mass spectrometer were automatically acquired with a customized sequence subroutine operated under Xcalibur software (v4.3, ThermoFisher) 47,49 . The resulting MDMS-SL data were analyzed and visualized in R (v3.6.0) and GraphPad Prism (v8.3.1). PC phosphatidylcholine, PI phosphatidylinositol, PE phosphatidylethanolamine, PS phosphatidylserine, SM sphingomyelin, LPC lysophosphatidylcholine, LPE lysophosphatidylethanolamine, PG phosphatidylglycerol, and PA phosphatidic acid.

Liquid chromatography-mass spectrometry (LCMS) lipidomics. Phospholipids were extracted and separated as previously performed ${ }^{50}$. MS analysis of phospholipids was performed on a Q-Exactive hybrid-quadrupole-orbitrap mass spectrometer (Thermo Fisher Scientific) using a normal phase column (Luna $3 \mu \mathrm{m}$ Silica $100 \AA, 150 \times 2.0 \mathrm{~mm}$, (Phenomenex)) at a flow rate of $0.2 \mathrm{~mL} / \mathrm{min}$ on a Dionex Ultimate 3000 HPLC system (maintained at $35^{\circ} \mathrm{C}$ ). The analysis was performed using A and B gradient solvents containing $10 \mathrm{mM}$ ammonium acetate and $0.5 \%$ triethylamine $(\mathrm{A}-$ propanol:hexane:water $(285: 215: 5, \mathrm{v} / \mathrm{v} / \mathrm{v})$ and $\mathrm{B}-$ propanol:hexane:water $(285: 215: 40, \mathrm{v} / \mathrm{v} / \mathrm{v}))$. The column was eluted for $0.5 \mathrm{~min}$ isocratically at $25 \% \mathrm{~B}$, then from 0.5 to $6.5 \mathrm{~min}$ with a linear gradient from 25 to $40 \%$ solvent B, from $6.5-25$ min using a linear gradient of $40-55 \%$ solvent $B$, from $25-38$ min with a linear gradient of $55-70 \%$ solvent $B$, from $38-48$ min using a linear gradient of $70-100 \%$ solvent $\mathrm{B}$, then isocratically from $48-55 \mathrm{~min}$ at $100 \%$ solvent B followed by a return to initial conditions from $55-70$ min from 100 to $25 \%$ B. The column was then equilibrated at $25 \%$ B for an additional $5 \mathrm{~min}$. Analysis was performed in negative ion mode at a resolution of 140,000 for the full MS scan in a data-dependent mode. The scan range for MS analysis was $400-1800$ $\mathrm{m} / \mathrm{z}$ with a maximum injection time of $128 \mathrm{~ms}$ using 1 microscan. An isolation window of $1.0 \mathrm{Da}$ was set for the MS and MS2 scans. Capillary spray voltage was set at $3.5 \mathrm{kV}$ and capillary temperature was $320^{\circ} \mathrm{C}$. The S-lens Rf level was set to 60. The phospholipid content for each APOE isoform was normalized to the amount of APOE protein as determined by SDS NuPAGE. The analysis of the resulting LCMS data was performed and visualized in R (v3.6.0) and GraphPad Prism (v8.3.1). See lipid class abbreviations in prior section.

A $\beta$ oligomer preparation. $A \beta_{42}$ peptide (American Peptide Company), HiLyte Fluor $488-\mathrm{A} \beta_{42}$, or HiLyte Fluor $555-\mathrm{A} \beta_{42}$ (AnaSpec) were used for all $\mathrm{A} \beta$ oligomerizations and injections ${ }^{27}$. Under a fume hood, $0.1 \mathrm{mg} A \beta$ peptide was dissolved in ice cold 1,1,1,3,3,3-Hexafluoro-2-Propanol (HFIP, Fluka) then vortex for a few seconds. The solution was dried with a gentle stream of nitrogen to obtain a peptide film at the bottom of the vial. Prior to use, the film was resuspended in anhydrous dimethyl sulfoxide (DMSO) to form a $5 \mathrm{mM}$ solution, sonicated in a water bath for $10 \mathrm{~min}$ and diluted in sterile phosphate-buffered saline (PBS) to a final concentration of $100 \mu \mathrm{M}$. HiLyte ${ }^{\text {wa }}$ Fluor 488 - or $555-\mathrm{A} \beta$ or $\mathrm{A} \beta_{42}$ peptide and E3 or E4 native particles were combined, vortexed, held under oligomer forming conditions (room temperature) for $24 \mathrm{~h}$ at a final concentration of $50 \mu \mathrm{M} \mathrm{A} \beta$ and 5 $\mu \mathrm{M} A P O E$ and stored at $-20^{\circ} \mathrm{C}$ until use (abbreviated $\mathrm{A} \beta \mathrm{E} 3$ or $\mathrm{A} \beta \mathrm{E} 4$ ). The same molar concentration (10:1) of scrambled $\mathrm{A} \beta$ (AnaSpec) was dissolved in vehicle, combined with isolated E3 or E4 particles, held under oligomer forming conditions and stored at $-20^{\circ} \mathrm{C}$ until use (abbreviated scrA $\beta E 3$ or scrA $\beta E 4$ ). scrA $\beta E 3$ or scrA $\beta E 4$ was utilized as negative controls throughout the subsequent experiments. Furthermore, $A \beta_{42}$ peptide and scrambled $A \beta$ was incubated with PBS vehicle as negative control for APOE particles.

\section{A $\beta$ oligomer characterization}

Western blot. A $\beta$ oligomers were examined using western blotting, proteins were resolved on 4-12\% Bis-Tris gels (Invitrogen) and transferred onto nitrocellulose membranes. These membranes were probed with 6E10 antibody (Biolegend, 1:2000) and anti-APOE antibody (Millipore, 1:4000) with immunoreactive signals visualized using enhanced chemiluminescence.

Electron microscopy. For electron microscopy, $5 \mu \mathrm{L}$ of each sample was placed on a freshly glow-discharged carbon-coated grid, adsorbed for $2 \mathrm{~min}$ and excess solution was blotted using filter paper ${ }^{27}$. The grid was washed with deionized water before staining with $5 \mu \mathrm{L}$ of freshly filtered uranyl acetate solution $(1 \%, w / v)$ for $15 \mathrm{~s}$. Excess stain was blotted and the grid was allowed to air-dry. Grids were imaged on a Tecnai T12 microscope (FEI) operating at $120 \mathrm{kV}$ and $\times 30,000$ magnification and equipped with an UltraScan 1000 CCD camera (Gatan) with post column magnification of $1.4 \mathrm{x}$.

Guide cannula implantation. To examine the cognitive effects in WT male mice (3 month), A $\beta$ oligomers co-incubated with native APOE lipid particles were infused directly into the brain through implanted guide cannulas ${ }^{27}$. Following anesthesia with isoflurane, the head was shaven and sterilized with two separate povidone-iodine-alcohol washes. A 50\% mixture of bupivacaine and lidocaine was applied to the surgical site and ophthalmic ointment applied to the eyes. The head was leveled in a stereotaxic frame and an incision made exposing the dorsal aspect of the skull. Two holes were drilled into the skull (coordinates: $\mathrm{AP}=-2.46 \mathrm{~mm}$, $\mathrm{L}= \pm 1.50 \mathrm{~mm}$ ) and 26-gauge guide cannulas (Plastics One) were lowered to a depth of $1.0 \mathrm{~mm}$. Cannulas were fixed to the skull with acrylic dental cement attached to two bone anchoring screws and the surgical opening sutured closed. After suturing, animals were administered buprenorphine and sterile saline, body temperature was maintained until sterna recumbence and animals were allowed to recover for 8 days prior to the start of behavioral testing.

Behavioral testing. All stages of behavioral testing were performed at the same time of the day (during the light phase), ensuring $24 \mathrm{~h}$ between each phase of testing. Fear conditioning was started $24 \mathrm{~h}$ after the completion of NOR. Prior to behavioral testing, mice were placed into individual containers, taken to the behavioral testing room and handled for $3 \mathrm{~min}$ for three consecutive days to reduce anxiety. Thirty minutes before a training stage, animals received an infusion of $A \beta$ oligomer co-incubated with either E3 or E4 (Fig. 2a-d). Scrambled A $\beta$ coincubated with either E3 or E4 was used as a negative control. Mice were randomly assigned to either the $\mathrm{A} \beta \mathrm{E} 3, \mathrm{~A} \beta \mathrm{E} 4$, scrA $\mathrm{BE} 3$, or scrA $\beta \mathrm{E} 4$ group. The dummy cannulas were removed and infusion cannulas, attached to microsyringe pump by polyethylene tubing, were placed in the guide cannula. $A \beta$ oligomer (final volume of $1 \mu \mathrm{L}$ per hemisphere) was infused over $1 \mathrm{~min}$, the cannulas were left in place for 1 min to allow for diffusion of the sample and finally dummy cannulas replaced. Each animal received five infusions of $A \beta$ oligomer starting on day 2 of NOR until the completion of behavioral testing (day 6). Between behavioral trials, the paradigms were cleaned with $70 \%$ ethanol to eliminate any olfactory cues. Performance 
was recorded and scored using ANY-maze software (Stoelting Co.) during all phases of testing.

Novel object recognition. NOR was performed over three consecutive days ${ }^{24}$. On day 1 , habituation phase, each animal was allowed to freely explore an open arena $(40 \mathrm{~cm} \times 40 \mathrm{~cm} \times 30 \mathrm{~cm}$ white plastic box) for two 5 min trials with a $5 \mathrm{~min}$ intertrial interval. On day 2, familiarization phase, each animal was returned to the arena containing two identical objects (tower of $\mathrm{LEGO}^{\circ}$ bricks) located in opposite diagonal corners for two 5 min trials separated with a 5 min intertrial interval. On day 3 , testing phase, the animal was returned to the arena with two objects in the same positions as previously, but one object was replaced with a novel object (metal bolt and nut of similar size). Mice were allowed to explore the two objects for 10 $\mathrm{min}$. The exploration of both objects was defined as the mouse sniffing or interacting while facing an object within $3 \mathrm{~cm}$. Mice were consistently placed into the middle of the arena facing the posterior wall to prevent any object preference. The percent exploration, an indicator of recognition memory, was determined by dividing the time exploring the novel object by the total time exploring both objects. Locomotor activity was assessed by measuring the total distance traveled in the open field during day 1 of testing.

Contextual and cued fear conditioning. Contextual and cued fear conditioning (CCFC) was performed over three consecutive days ${ }^{24}$. On day 1 , training phase, mice were placed in a conditioning chamber (Stoelting Co.) for $5.5 \mathrm{~min}$. The first 2 min were silent, allowing the mouse to acclimate to the chamber; this was followed by a $30 \mathrm{~s}$ tone $(2800 \mathrm{~Hz}$; Intensity $85 \mathrm{~dB}$, conditioned stimulus (CS)) ending in a $2 \mathrm{~s}$ foot shock $(0.7 \mathrm{~mA}$, unconditioned stimulus (US)) through the floor of the conditioning chamber. The process was repeated one more time and ended with $30 \mathrm{~s}$ of re-acclimation. On day 2, contextual phase, mice were placed in the same conditioning chamber for $5 \mathrm{~min}$ with no tone or shock administered, to measure contextual fear conditioning. On day 3 , the gray walls of the chamber were replaced with black and white striped walls to introduce a novel environment for assessing cued fear conditioning. Mice were placed in the conditioning chamber for $5 \mathrm{~min}$. After the first $2 \mathrm{~min}$ of silence, the tone was administered for $3 \mathrm{~min}$, to measure cued fear conditioning. Freezing time was defined as the absence of movement except for respiration and calculated as percent freezing of the total time in the chamber during each phase of testing.

Cortical infusion. WT male mice (3 month) received cortical infusions of unlabeled $A \beta E 3$ or $A \beta E 4$. In a separate cohort of $\mathrm{Cx} 3 \mathrm{cr} 1^{\mathrm{GFP}}$, WT, and Trem $2^{\mathrm{ko}}$ male mice ( 3 month), HiLyte ${ }^{\mathrm{m}}$ Fluor 555 -labeled $\mathrm{A} \beta$ combined with native E3 and E4 particles was infused into the cortex. Mice were randomly assigned to either the scrambled $A \beta, A \beta$ alone, $A \beta E 3$, or $A \beta E 4$ group. For the bilateral infusion, a 28gauge infusion cannula (Plastics One) was connected to a $10 \mu \mathrm{L}$ glass syringe (Hamilton) with vinyl tubing and placed in a micro syringe pump. Following anesthesia with isoflurane, the head was shaven and sterilized with two separate povidone-iodine-alcohol washes. A 50\% mixture of bupivacaine and lidocaine was applied to the surgical site and ophthalmic ointment applied to the eyes. The head was leveled in a stereotaxic frame and an incision made, exposing the dorsal aspect of the skull. Four holes were drilled into the skull (coordinates: $\mathrm{AP}=-1$ and -2.5 $\mathrm{mm}, \mathrm{L}= \pm 2.0 \mathrm{~mm})$ and the infusion cannula was lowered into the cortex (DV = $-1.0 \mathrm{~mm}$ ). At each infusion site, $2 \mu \mathrm{L}$ of $\mathrm{A} \beta$ preparation was infused at a rate of 0.5 $\mu \mathrm{L} / \mathrm{min}$ and the cannula remained in place for 4 min following the infusion. Following the infusions, the surgical opening was sutured closed, animals were administered buprenorphine and sterile saline and placed on a heating pad until fully recovered.

Animal tissue processing. Four or $24 \mathrm{~h}$ following the cortical infusion, mice were perfused and tissues collected. Mice were anesthetized with Avertin $(250 \mathrm{mg} / \mathrm{kg}$ of body weight, i.p.) and transcardially perfused with $25 \mathrm{~mL}$ of cold $0.1 \mathrm{M} \mathrm{PBS}, \mathrm{pH}$ 7.4. For Cx3 $31^{\mathrm{GFP}}$ and Trem $2^{\mathrm{ko}}$ mice used for FACS, flow cytometry and single cell RNA-seq the infusion sites in both hemispheres were excised for cellular isolation as described below. For WT mice, one hemisphere was drop fixed in $4 \%$ phosphate-buffered paraformaldehyde at $4{ }^{\circ} \mathrm{C}$ for $48 \mathrm{~h}$ before storage in $30 \%$ sucrose. The other hemisphere was used for cellular isolation as described below. Fixed hemibrains were mounted in O.C.T. and cut in the coronal plane at $30 \mu \mathrm{m}$ sections using a frozen cryotome (Thermo Scientific) and stored in glycol-based cryoprotectant at $-20^{\circ} \mathrm{C}$ until histological staining. Prior to staining, sections containing the infusion site were selected under a dissection microscope (Olympus). In a separate cohort of APP/E3 and APP/E4 mice (14 month) CSF was collected prior to perfusion. Once anesthetized a small incision was made exposing the foramen magnum, a $28 \mathrm{~g} \mathrm{1/2"} \mathrm{needle} \mathrm{was} \mathrm{used} \mathrm{to} \mathrm{puncture} \mathrm{a} \mathrm{hole} \mathrm{at} \mathrm{the} \mathrm{base} \mathrm{of}$ the skull and a capillary tube was applied for CSF withdraw.

Immunohistochemistry. To characterize changes in activated microglia in the vicinity of the infusion site, a series of six brain sections from each animal was immunostained with anti-IBA1 antibody (WAKO). Free-floating sections were washed, followed by antigen retrieval in sodium citrate buffer at $80^{\circ} \mathrm{C}$ for $60 \mathrm{~min}$, blocked in Normal Donkey Serum (Jackson Lab, 5\%) for $1 \mathrm{~h}$, and finally incubated in IBA1 antibody $(1: 1000)$ overnight at $4{ }^{\circ} \mathrm{C}$. Sections were washed and transferred into secondary donkey anti-rabbit Alexa 594 antibody (Invitrogen, 1:250) for $1 \mathrm{~h}$, before being washed, mounted on superfrost plus slides and coverslipped. Fluorescent confocal images of the infusion site were taken using a Nikon A1 confocal microscope at 60x magnification with $1.0 \mu \mathrm{m}$ step size. Four sequential images were captured flanking either side of the infusion site. Analysis was performed as in Marsh et $\mathrm{al}^{51}$. with modifications. The FilamentTracer module (Imaris, version 7.1.1, Bitplane) was utilized to trace processes of cortical IBA1-positive microglia and determine process length and number of branch points as indicators of microglial morphology complexity. Using the 3D mapped filament tracings, we oriented all microglia so their central axis was positioned facing the infusion site and generated a heatmap depicting the location of microglia from an experimental group utilizing the ImageJ - heatmap from image stack plugin (v1.53e, National Institutes of Health). The number of overlapping microglial processes increases the pixel saturation metric. The percent of all processes which occupy space in each of the four quadrants was used to determine the percent coverage of that quadrant.

A second series of six brain sections around the infusion site was used for F4/80 immunostaining. First, we quenched endogenous peroxidases with $0.3 \%$ hydrogen peroxide, tissues were blocked in 3\% normal goat serum (Vector), then blocked for endogenous avidin and biotin. Sections were incubated in F4/80 antibody (Abcam, 1:500) overnight at $4{ }^{\circ} \mathrm{C}$. Sections were washed and transferred into secondary biotinylated anti-rat antibody (Vector, 1:250) for $90 \mathrm{~min}$ before being washed and subsequently developed using the Vector ABC kit and DAB substrate kit (Vector). Sections were mounted onto superfrost plus slides and coverslipped. Bright-field images were taken using a Nikon Eclipse 90i microscope at 20x magnification encompassing the infusion site. Image intensity threshold was established to detect the F4/80 staining compared to background using NIS Elements software (Nikon Instruments Inc.) and values were represented as the area of staining normalized to total image area or percentage of area covered.

In a separate cohort of APP/E3 and APP/E4 mice (6 month) free floating section were stained for DAPI and IBA1 as above and six confocal images of the cortex were captured (Nikon A1; 40x, $1.0 \mu \mathrm{m}$ step size). The morphology of IBAl positive microglia was determined by seed point labeling of cell bodies and dendrites as described above utilizing Imaris. An adjacent series of brain tissue was used for Thioflavin S (ThioS) staining. Sections were mounted onto slides, washed in PBS, and stained with $0.02 \%$ ThioS (Sigma, USA) in PBS for $10 \mathrm{~min}$. Next, sections were differentiated in 50\% ethanol for $2 \mathrm{~min}$. After a final wash in PBS, slides were coverslipped. Fluorescent images were taken using a Nikon Eclipse 90i microscope at $4 \mathrm{X}$ magnification. To quantify plaque pathology, two separate regions of interest (ROI) were drawn around the cortex and hippocampus for each section and an image intensity threshold was established to detect the stained plaques compared to the background using NIS Elements software (Nikon Instruments Inc., USA). ThioS staining values were represented as the area of staining normalized to ROI area or percentage of area covered by ThioS stain. Microglia process length was correlated to percent ThioS staining with nonparametric correlation analysis.

In vivo plaque labeling. $\mathrm{X} 04(10 \mathrm{mg} / \mathrm{kg})$ was administered intraperitoneally to $\mathrm{APP} / \mathrm{E} 3$ and APP/E4 mice (3-5 month) and sacrificed 30 days later for in vivo labeling of dense core amyloid plaques in brain ${ }^{24}$. Tissue was processed and freefloating sections were immunostained with anti-IBA1 as above and labeled with goat anti-rabbit Dylight 488 (Di-1488 Vector Labs, 1:250) as described above. Stained tissues were imaged on an Olympus FV1000 confocal microscope at 60x ( $1.5 \mu \mathrm{m}$ step size); with plaques selected at least $50 \mu \mathrm{m}$ from the tissue edge and at least $50 \mu \mathrm{m}$ away from other plaques, with an even representation of all plaque sizes and composition across groups. Images were loaded into the Imaris (v9.3.1) environment and voxels less than 500 intensity were removed to reduce background noise. Surfaces were then generated for both channels and coverage determined using the "surface-surface contact area" XTension. Sum of colocalized voxels divided by the total surface area of the plaque generated the percent microglia coverage. An adjacent series of brain tissue was used for Thioflavin S (ThioS) staining and analysis as described above. Percent microglia plaque coverage was correlated to percent ThioS staining with simple linear regression.

Magnetic-activated cell sorting (MACS). For WT mice infused with unlabeled $\mathrm{A} \beta$, we isolated microglial and neuronal cellular populations utilizing MACS column-based protocols according to the manufacturer's instructions (Miltenyi Biotec). First, the tissue was dissociated utilizing the Neural Tissue Dissociation kit (Miltenyi Biotec), a gentle two-step enzymatic dissociation (papain and trypsin) that yields a high number of viable cells. Briefly, the cortical tissue was enzymatically and mechanically lysed at $37^{\circ} \mathrm{C}$ for $35 \mathrm{~min}$ to obtain a cell suspension. Then the suspension was passed through a $40 \mu \mathrm{m}$ cell strainer to remove debris and ensure a single-cell suspension. After a $10 \mathrm{~min}$ centrifugation at $300 \mathrm{xg}$, the pellet was gently resuspended with ice-cold PBS $+0.5 \%$ BSA buffer and incubate for 15 min at $4{ }^{\circ} \mathrm{C}$ with Myelin Removal Beads II (Miltenyi Biotec). After washing with $\mathrm{PBS}+0.5 \% \mathrm{BSA}$ and $10 \mathrm{~min}$ centrifugation at $300 \mathrm{xg}$, the pellet was resuspended with PBS $+0.5 \%$ BSA. The single-cell suspension was applied to a column placed on a magnetic stand (LS column and magnets from Miltenyi Biotec) to deplete the myelin fragments by magnetic separation and washed with PBS $+0.5 \%$ BSA. Finally, the cells were centrifuged for $5 \mathrm{~min}$ at $300 \mathrm{~g}$ and the pellet was resuspended with $1 \mathrm{~mL}$ of PBS $+0.5 \%$ BSA. After obtaining a myelin-free single-cell suspension, 
the microglia were labeled with anti-CD11b immunomagnetic beads and isolated using magnetic columns (Miltenyi Biotec). To isolate the neurons, the cell suspension depleted of microglia cells was incubated with a cocktail of antibodies from the Neuron Isolation Kit (Miltenyi Biotec). Non-neuronal cells like astrocytes, oligodendrocytes, endothelial cells, or fibroblasts were magnetically labeled and depleted through the magnetic columns; therefore, neurons were isolated by negative selection. To confirm the purity of isolated microglia, we performed RTqPCR with P2ry12 and Tmem119, microglia-specific markers (Supplementary Table 2). To further ensure microglia and neuron purity when using MACS, following sequencing and alignment, all genes with low expression (average raw read count $<30.9$ ) were removed from further analysis.

\section{Fluorescence-activated cell sorting (FACS) and flow cytometry. For the} Cx3crl GFP mice infused with HiLyte ${ }^{m}$ Fluor 555-labeled A $\beta$, microglia were isolated utilizing FACS. After removing the cerebellum, subcortical area and olfactory bulbs, cortical tissue within $1 \mathrm{~mm}$ of either side of the infusion site was processed into a single-cell suspension using the Neural Tissue Dissociation kit followed by the Myelin Removal Beads II (Miltenyi Biotec) as described above. For WT mice, after the myelin removal steps the pellet were resuspended with $100 \mu \mathrm{l}$ of PBS and the microglia cells were labeled with $5 \mu$ of anti-mouse/human CD11b antibody (clone M1/70) conjugated with Alexa Fluor 647 (BioLegend, 1:100) for $20 \mathrm{~min}$ on ice. All cells were then washed and prepared with $1 \mathrm{~mL}$ of PBS $+0.5 \%$ BSA for FACS sorting.

A bio-contained BD FACSAria" III sorter (BD Biosciences) was used to sort microglia cells for the two experimental populations. First, live cells were separated from the debris according to their forward scatter and side scatter properties and a second gate was used on individual cells only. The GFP fluorescence was detected with a $525 \mathrm{~nm}$ filter ( $488 \mathrm{~nm}$ laser) for the microglia isolated from the $\mathrm{Cx} 3 \mathrm{cr} 1{ }^{\mathrm{GFP}}$ mice, the CD11b/APC fluorescence was collected with a $668 \mathrm{~nm}$ filter ( $647 \mathrm{~nm}$ laser) for the WT mice and the 555-labeled A $\beta$ fluorescence was detected with a $613 \mathrm{~nm}$ filter ( $555 \mathrm{~nm}$ laser). About 2000 microglia with high GFP signal but low 555labeled $A \beta$ signal, were considered unexposed to $A \beta$ and termed single-positive cells $($ single + ). About 2000 microglia cells with a high signal for both labels (GFP and 555-labeled $A \beta$ ) were considered exposed to $A \beta$ and termed dual-positive cells (dual+). The single + and dual+ microglial populations were sorted into $1.5 \mathrm{~mL}$ tubes containing $350 \mu \mathrm{L}$ of RLT lysis buffer from the RNeasy Micro kit (Qiagen) and $3.5 \mu \mathrm{L}$ of 2-mercaptoethanol. After the FACS sorting, the bio-contained BD FACSAria ${ }^{\text {"m }}$ III sorter (BD Attovision ${ }^{\mathrm{TM}}$ software, BD biosciences) was used with the same gates to count and estimate the percentages of the total microglia population and single + and dual + microglia population in a total of $1,000,000$ live cells.

RNA isolation and RNA-sequencing. RNA was isolated from the MACS-isolated microglia and neurons and FACS single + and dual+ microglia following the manufacturer's instructions for the RNeasy Mini kit and RNeasy Micro kit (Qiagen), respectively. After RNA isolation, concentration was measured with a Qubit 3.0 Fluorometer (Thermofisher) before quality assessment with the 2100 Bioanalyzer instrument (Agilent Technologies). Sequencing libraries for MACS-isolated cells were generated using mRNA Library Prep Reagent set (Illumina) according to the manufacturer's instructions ${ }^{52}$. The total RNA from sorted cells was fragmented and converted into cDNA using the SMART-Seq ${ }^{\circledR}$ v4 Ultra Low Input RNA Kit for Sequencing (Takara Bio). Briefly, a minimum of $10 \mathrm{pg}$ of purified total RNA was used to perform a first-strand cDNA synthesis in a PCR clean workstation. After amplification by LD PCR and purification using the Agencourt AMPure XP beads (Beckman Coulter), $1 \mu \mathrm{L}$ of the amplified cDNA was used for validation using the Agilent 2100 Bioanalyzer on a High Sensitivity DNA chip. One nanogram of the cDNA was used with the Nextera DNA Library Preparation Kit (Illumina) to prepare the libraries. After tagmentation, the cDNA was linked with two different index adapters from the Nextera XT Index Kit (Illumina) during the amplification by PCR. Each sample received a unique combination of two specific index/barcodes during the library generation. Finally, the libraries were purified using Agencourt AMPure XP beads that provide a size selection to remove short library fragments. Again, $1 \mu \mathrm{L}$ of the libraries was used for validation using the Agilent 2100 Bioanalyzer on a High Sensitivity DNA chip. After preparation of the libraries, sequencing was performed by the Next Generation Sequencing Center (University of Pennsylvania, https://ngsc.med.upenn.edu/) on HiSeq 2500 machine.

mRNA-seq data processing. Following initial processing and quality control, the sequencing data was aligned to the mouse genome $\mathrm{mm} 10$ using Subread/featureCounts (v1.5.3; https://sourceforge.net/projects/subread/files/subread-1.5.3/) with an average read depth of $21,554,242$ successfully aligned reads. Statistical analysis was carried out using Rsubread (v1.34.2; https://bioconductor.org/packages/ release/bioc/html/Rsubread.html), DEseq2 (1.24.0; https://bioconductor.org/ packages/release/bioc/html/DESeq2.html), and edgeR (v3.26.5; https:// bioconductor.org/packages/release/bioc/html/edgeR.html), all in the R environment (v3.6.0; https://www.r-project.org/). Functional annotation clustering was performed using the Database for Annotation, Visualization and Integrated Discovery (DAVID v6.8, https://david.ncifcrf.gov). All GO terms were considered significant if $p<0.05$ following corrections for multiple comparisons using the Benjamini-Hochberg method to control the FDR. $t$-Distributed stochastic neigh bor embedding (tSNE) plots were generated to visualize patterns of similarity between groups. To generate the tSNE, normalized data from all genes in the analysis were submitted and the perplexity set as high as possible given the total number of samples in the analysis with the theta set to 0.5 .

Single-cell isolation and library creation. Separate biological replicates from $A \beta E 3$ or $A \beta E 4$ infused WT and Trem $2^{\text {ko }}$ mice were used for scRNA-seq and infused as described above. Twenty-four hours after the injections mice were perfused with PBS. Microglia from non-injected WT mice were used as negative control. After the brain extraction, cortical tissue around the injection site were excised using adult mouse brain matrix at $1 \mathrm{~mm}$ before and after the injection site. The brain tissue was then dissociated according to the manufacturer's instructions (Miltenyi Biotec) as described above. CD11b-purified cells were resuspended in $0.04 \%$ BSA/PBS solution and passed through a $40-\mu \mathrm{m}$ nylon and a $20-\mu \mathrm{m}$ nylon cell strainer to obtain single cell suspension. The cell viability and number were assessed using a Countess II FL Automated Cell Counter (ThermoFisher) with a Live/Dead viability/Cytotoxicity kit (Invitrogen). The single cell suspension with $>90 \%$ viability was immediately loaded onto the Chromium controller (10x Genomics) in order to capture $~ 5000$ cells per sample. Libraries were generated with Chromium single cell $3^{\prime}$ chip kit $\mathrm{v}$ for scRNA-seq (10x Genomics) according to the manufacturer's instructions and then the quality assessed using Agilent Bioanalyzer 2100 . Sequencing was performed using NovaSeq (Illumina) by MedGenome Inc. with a custom sequencing setting ( 28 bp for Read1 and 91 bp for Read2) to obtain an average sequencing depth of $45 \mathrm{~K}$ reads/cell.

scRNA-seq data processing. After libraries were sequenced and quality control was performed, samples were aligned to the $\mathrm{mm} 10$ mouse reference genome using the Cell Ranger 3.0.1 pipeline (https://support.10xgenomics.com/single-cell-geneexpression/software/pipelines/latest/what-is-cell-ranger). Each sample was then aggregated using the cellranger aggr function to produce a raw UMI count matrix containing the number of reads for genes in each cell per sample. The expression matrix was then loaded into $\mathrm{R}$ for further analysis and visualization using Seurat v3.0.2 ${ }^{53}$. Genes expressed in fewer than 200 cells were excluded from further analysis. The number of genes expressed in each cell (nGene), the number of reads in each cell (nCount), and the percentage of reads mapped to mitochondrial genes (percent.mito) were obtained for each cell. Cells were then filtered to reduce the potential of including doublet and low-quality cells using the following criteria: $200<$ nGene $<8500 ; 500<$ nCount $<90,000$; and percent.mito $<25 \%$. Feature counts were normalized using LogNormalize method with a scale factor of 10,000; and the effects of nGene, nCount, and percent.mito were regressed out using the ScaleData method. A shared nearest neighbor (SNN) graph was constructed using FindNeighbors function with default parameters. Using the Louvain algorithm implemented in FindClusters function with a clustering resolution of 0.4 and the first 60 PCs, we identified 20 clusters. To determine the cell types, present in each cluster, we examined the expression levels of cell type specific markers across each cluster and identified clusters containing unique populations of microglia, neurons, astrocytes, vascular cells, ependymal cells, and myeloid cells. We used FindAllMarkers function to identify genes that act as markers for each cluster, using the Wilcoxon rank-sum test. A gene was considered the marker of a cluster if it had a Bonferroni-adjusted $p$ value $<0.01$ and an average log fold change $>0.1$. The data were then filtered to contain clusters containing only microglia and cells with a normalized expression <1 for Aldoc, F13a1, Mylk, Meg3, Gfap, Thy1, Slc17a7,

Olfm1, Aqp4, Dlx1, Olig2, Foxj1, Nkg7, Vtn, Flt1, Acta2, and S100a9 were removed, resulting in the retention of 39,898 microglial cells. To control for the potential of batch effect we regressed out the percentage of UMI counts in each cell belonging to several sex-linked genes: $D d x 3 y, E i f 2 s 3 y, X i s t, K d m 5 d$, and Uty. Using a clustering resolution of 0.15 and the first 50 PCs, we identified six separate clusters. The data were further filtered to contain only cells from injected samples, resulting in the retention of 36,244 microglial cells. After reclustering with a resolution of 0.1 and the first 50 PCs, we identified five clusters. To perform differential expression analysis, we used Seurat's FindMarkers function and performed Wilcoxon ranksum tests. A gene was considered differentially expressed if it had a Bonferronicorrected $p$ value $<0.05$ and a natural $\log$ fold change $(\log F C)>0.1$. Seurat data objects were reformatted for pseudotime analysis using the SingleCellExperiment package and trajectory analysis was performed using Slingshot ${ }^{54}$ establishing cluster $1 \mathrm{a}$ as the starting cluster and clusters 2,3 , and 4 as terminal clusters.

Fluorescence in situ hybridization (FISH). In a separate infused cohort, mice were perfused, tissue fixed, and sectioned as documented above. RNAscope experiments were performed using the Multiplex Fluorescent Reagent kit v2 (Advanced Cell Diagnostics) following the manufacturer's recommendations with minor adjustments. Six freshly sectioned tissues per animal were mounted onto superfrost plus slides within a $0.75^{\prime \prime} \mathrm{x} 0.75^{\prime \prime}$ square and baked at $60^{\circ} \mathrm{C}$ for $60 \mathrm{~min}$. Slides were dehydrated using a series of ethanol dilution steps, then submerged in target retrieval reagent at $100^{\circ} \mathrm{C}$ for $10 \mathrm{~min}$. Protease digestion was performed at $40^{\circ} \mathrm{C}$ for $30 \mathrm{~min}$ using Protease III and probe hybridization was carried out at $40^{\circ} \mathrm{C}$ for $2 \mathrm{~h}$. We used probe sets available from ACD for Adgrel and Tmem 119 . Following the amplification steps, the sections were counterstained with DAPI and coverslipped. Imaging was carried out using a Nikon Eclipse 90i microscope at 20X magnification with tiled imaging of the entire infusion site and analyzed using NIS 
Elements software (Nikon Instruments Inc.). Four $200 \mu \mathrm{m}$ x $200 \mu \mathrm{m}$ ROIs were drawn and a threshold established for each probe to determine the area of puncta coverage. The four ROI were averaged together to create one data point per infusion site representing the average area of puncta coverage within $200 \mu \mathrm{m}$ of the edge of the infusion site.

Two-photon imaging and statistical analysis. We used $\mathrm{Cx} 3 \mathrm{crl}{ }^{\mathrm{GFP}}$ male mice ( 3 month) to determine the microglial response of $A \beta E 3$ or $A \beta E 4$ utilizing twophoton imaging ${ }^{55}$. Briefly, the animals were anesthetized I.P. with a cocktail of ketamine/xylazine $(75 / 10 \mathrm{mg} / \mathrm{kg}$ ) and placed in a stereotaxic frame. Body temperature was maintained at $37.6^{\circ} \mathrm{C}$. The skin above the skull was removed and a well of $2 \mathrm{~cm}$ diameter was built over both hemispheres with a light-curable cement (Composite Flowable) to hold a saline immersion for the microscope objective. The craniotomy was conducted with a high-speed dental drill over the parietal cortex bilaterally. The craniotomy site was regularly flushed with saline to wash out bone fragments and prevent thermal damage. The exposed cortex was injected with HiLyte ${ }^{\text {ma }}$ Fluor 555-labeled A $\beta$ (Anaspec) combined with native E3 or E4 particles preincubated for $24 \mathrm{~h}$. The injection was performed at an approximate depth of $300 \mu \mathrm{m}$ below the surface of the cortex with a borosilicate glass capillary tube (WPI) pulled with a two-stage vertical pipette puller (Narishige) to a tip diameter of $\sim 10 \mu \mathrm{m}$ and coupled with flexible tubing to a picospritzer microinjection dispense system (Parker). Mice were randomly assigned to either the $\mathrm{A} \beta \mathrm{E} 3$ or $\mathrm{A} \beta \mathrm{E} 4$ group. After the injection, the mice were moved under the two-photon microscope within $5 \mathrm{~min}$ and anesthesia was maintained with $40 \mathrm{mg} / \mathrm{ml} / \mathrm{h}$ ketamine.

In vivo imaging was conducted with an Ultima IV two-photon laser scanning microscope (Bruker) with an InSight DS Laser system (Spectra-Physics) tuned at a wavelength of $920 \mathrm{~nm}$. Two channels were collected red (595/50) and green (525/ $50 \mathrm{~nm}$ ) to visualize the 555-labeled $A \beta$ and microglia, respectively. Images were collected with 16x Nikon objective lens on Prairie View software using a time series of 11 slices with $1024 \times 1024$ matrix size, dwell time $4.4 \mu$ s and $3 \mu \mathrm{m}$ step size. The effective in-plane resolution was $0.4 \mu \mathrm{m}$ per pixel. The Z-stack images were collected in Galvo acquisition mode such that each Z-stack was collected every minute for a total duration of $80 \mathrm{~min}$.

For each animal, Z-stack images were analyzed to quantify the microglial approach and microglial injection site coverage over time. Initially, the data were loaded in ImageJ (National Institutes of Health) and a rigid-body co-registration with the StackReg plugin was used to account for global temporal drifts of the field of view. The Z-stacks were transformed to sum-based XY projections by linear interpolation using the 3D Project function, allowing for time series analysis of specific ROI. We generated the panels illustrating cell displacement from those datasets by assigning red to the beginning of the time series $(t=0)$ and green to subsequent timepoints (10 or $80 \mathrm{~min}$ ). Then, the two color-coded images were merged in ImageJ to reveal spatial details, with red showing the original location, green for the new cellular location after a particular segment of time has elapsed (0-10 $\mathrm{min}$ or $0-80 \mathrm{~min})$, and yellow at the regions where the two channels overlap. The 555-labeled $A \beta$ infusion site was shown in white to display the increasing coverage as cell processes approach. The time series were also saved as movies presented in the supplementary material.

The datasets were then converted to mat structures and all subsequent analyses were performed in MATLAB (R2019a, Math Works), using standard image processing and statistical functions. Briefly, the red channel showing the infusion site was thresholded using the Otsu's method with the multithresh Matlab function. The resulting images were binarized and small features outside of the infusion site were removed using the bwareaopen function. The mask was then dilated to smooth the borders and account for the lower contrast of the diffusion gradient around the borders. The percent coverage by the microglial processes over time was then computed as the increase of the green channel intensity above 1.5 times the standard deviation baseline of the green channel within the binary mask.

The mean distance of the cell processes to the infusion site over time was computed by eroding the binary mask described above and computing the Euclidean distance transformation of the full binary image using the bwdist function. Then, each pixel in the green channel over 1.5 standard deviations of the intensity was mapped with that transformation and assigned a number that is the distance between that pixel and the edge of the infusion site forming progressive concentric level sets with increasing proximity to the infusion site. The change of this Euclidean distance over time was converted from pixels to microns using the effective in-plane resolution of the imaging data and plotted as a time series.

To verify $A \beta$ biodistribution, Z-stack images had two color channels-red channel $(595 / 50 \mathrm{~nm})$ to capture the A $\beta$ + APOE infusion and green channel (525/ $50 \mathrm{~nm}$ ) to establish the initial positions of microglia within the infusion volume. The in-plane matrix size was $1024 \times 1024$ pixels with an effective resolution of 0.8 $\mu /$ pixel. We used a step size of $5 \mu$ along the $z$ direction across the cortical thickness, delivering an effective tissue volume of $3.251 \mu^{3}$ per pixel. Each Z-stack was collected across the full depth of the infusion site with an average stack size of 30 slices and image analysis was performed in MATLAB. In order to quantify the three-dimensional distribution of the infusion, we converted the 16-bit red channel to binary matrix based on global foreground mean threshold (Otsu's method), eroded all small noise regions not connected to the infusion site and rendered the surface to verify consistent shape based on diffusion from the injection tip. We used the volume-per-pixel value $\left(3.251 \mu^{3}\right)$ to calculate the total infusion volume based on the number of non-zero matrix elements within the three-dimensional binary mask.

Primary microglia cell culture. Primary microglial culture and A $\beta$ uptake assay were performed using WT, E3, E4, Trem2 $2^{\mathrm{ko}}, \mathrm{E} 3 /$ Trem $2^{\mathrm{ko}}$, and E4/Trem $2^{\mathrm{ko}}$ pups (1-3 days old $)^{44,45}$. The cortices and hippocampi were mechanically dissociated using a sterile Pasteur pipette. The dissociated cells were plated in $75 \mathrm{~cm}^{2}$ flasks with DMEM/F12 medium (Thermo Fisher) containing 10\% FBS at DIV0. Twenty-four hours after plating (DIV1), the media was replaced to remove debris. Microglia were collected by tapping at DIV14 and plated on $0.01 \%$ poly-L-lysine (Sigma) coated 12 $\mathrm{mm}$ circular coverslips in 24-well plates with the density of 60,000 cells/well.

Twenty-four hours after plating, cells in the treatment groups were treated with 1 $\mu \mathrm{M}$ Hi-Lyte ${ }^{\mathrm{TM}}$ Fluor 488-labeled $\mathrm{A} \beta$ or Hi-Lyte ${ }^{\mathrm{TM}}$ Fluor 555-labeled A $\beta$ (Anaspec) at $37^{\circ} \mathrm{C}$ for $1 \mathrm{~h}$. Following the treatment, cells were washed three times with PBS, fixed in $4 \%$ PFA, and permeabilized with $0.2 \%$ Triton X-100 at room temperature Microglia were then labeled with Anti-IBA1 antibody (1:500) at $4{ }^{\circ} \mathrm{C}$ for $18 \mathrm{~h}$ and followed by $2 \mathrm{~h}$ incubation with horse anti-rabbit 594 secondary antibody (1:250) for 488-labeled $A \beta$ or donkey anti-rabbit 488 secondary antibody (1:250) for 555labeled $A \beta$. Microglia were stained with DAPI to visualize nuclei.

Fluorescent images of in vitro $A \beta$-treated microglia were taken on a Nikon Eclipse 90i microscope (20X magnification) and analyzed in NIS elements (Nikon Instruments Inc.). Exposure levels for each channel were consistent across all genotypes and samples. Images were thresholded to identify microglia, nuclei, and $A \beta$. To assess the $A \beta$ uptake in microglia, the percentage of microglia containing $A \beta$ was determined by dividing IBA $1+$ microglia colocalized with $A \beta$ signal by the total of IBA1 + microglia and averaged across all images in each genotype.

Quantification and statistical analysis. Sample sizes $(n)$ indicated in the figure legend 1 indicate number of separate pools of astrocyte conditioned media. Sample sizes $(n)$ indicated in the figure legend 2-7 correspond to the number of biological replicates analyzed. All researchers were blinded to experimental groups during the analysis. All results are reported as means \pm SEM.

Unless otherwise indicated, all statistical analyses were performed in GraphPad Prism (v 8.3.1), or in R ( $\mathrm{v} 3.6 .0$ ) and significance was determined as $p<0.05$. Number of experiments and statistical information are stated in the corresponding figure legends. In figures, asterisks denote statistical significance marked by ${ }^{*} p<$ $0.05 ; * * p<0.01 ; * * * p<0.001$.

Reporting summary. Further information on research design is available in the Nature Research Reporting Summary linked to this article.

\section{Data availability}

The bulk RNA-seq and single cell RNA-seq data in this study are available in the NCBI Gene Expression Omnibus (GEO) database under the accession number: GSE158962 (https://www.ncbi.nlm.nih.gov/geo/query/acc.cgi?acc=GSE158962). All of the sequencing datasets used in this study were processed using publicly available open-source software. The sequencing data was aligned to the mouse genome $\mathrm{mm} 10$ (https://bioconductor.org/ packages/release/data/annotation/html/org.Mm.eg.db.html). Functional annotation clustering was performed using the Database for Annotation, Visualization and Integrated Discovery (DAVID v6.8, https://david.ncifcrf.gov). Source data are provided with this paper. All data supporting the findings of this study are provided within the paper and its supplementary information. Additional information is available upon reasonable request to the authors. Source data are provided with this paper.

\section{Code availability}

Bulk RNA-seq and single cell RNA-seq data were processed using publicly available open-source software and standard pipelines were used, as described in the Methods. The Rsubread (v1.34.2), DEseq2 (v1.24.0), and edgeR (v3.26.5) were used for bulk RNA-seq data analysis. The Seurat (v3.0.2) and Slingshot (v1.8.0) were used for single cell RNAseq data analysis. All relevant code is available from the authors upon reasonable request.

\section{Received: 24 September 2020; Accepted: 14 May 2021;} Published online: 07 June 2021

\section{References}

1. Association, A. 2019 Alzheimer's disease facts and figures. Alzheimers Dement. 15, 321-387 (2019).

2. Corder, E. H. et al. Gene dose of apolipoprotein E type 4 allele and the risk of Alzheimer's disease in late onset families. Science 261, 921-923 (1993).

3. Saunders, A. M. et al. Association of apolipoprotein $\mathrm{E}$ allele epsilon 4 with lateonset familial and sporadic Alzheimer's disease. Neurology 43, 1467-1472 (1993).

4. Weisgraber, K. H. Apolipoprotein E distribution among human plasma lipoproteins: role of the cysteine-arginine interchange at residue 112. J. Lipid Res. 31, 1503-1511 (1990) 
5. Hatters, D. M., Peters-Libeu, C. A. \& Weisgraber, K. H. Apolipoprotein E structure: insights into function. Trends Biochem. Sci. 31, 445-454 (2006).

6. Lefterov, I. et al. APOE2 orchestrated differences in transcriptomic and lipidomic profiles of postmortem AD brain. Alzheimers Res. Ther. 11, 113-113 (2019)

7. Wolfe, C. M., Fitz, N. F., Nam, K. N., Lefterov, I. \& Koldamova, R. The role of APOE and TREM2 in Alzheimer's disease-current understanding and perspectives. Int. J. Molecular Sci. https://doi.org/10.3390/ijms20010081 (2018).

8. Koldamova, R., Fitz, N. F. \& Lefterov, I. ATP-binding cassette transporter A1: from metabolism to neurodegeneration. Neurobiol. Dis. 72 Pt A, 13-21 (2014).

9. DeMattos, R. B. et al. Purification and characterization of astrocyte-secreted apolipoprotein $\mathrm{E}$ and J-containing lipoproteins from wild-type and human apoE transgenic mice. Neurochem. Int. 39, 415-425 (2001).

10. $\mathrm{LaDu}, \mathrm{M}$. J. et al. Isoform-specific binding of apolipoprotein $\mathrm{E}$ to betaamyloid. J. Biol. Chem. 269, 23403-23406 (1994).

11. Deane, R. et al. apoE isoform-specific disruption of amyloid beta peptide clearance from mouse brain. J. Clin. Invest. 118, 4002-4013 (2008)

12. Jiang, Q. et al. ApoE promotes the proteolytic degradation of Abeta. Neuron 58, 681-693 (2008).

13. Fitz, N. F. et al. Abcal deficiency affects Alzheimer's disease-like phenotype in human ApoE4 but not in ApoE3-targeted replacement mice. J. Neurosci. 32, 13125-13136 (2012)

14. Holtzman, D. M. et al. Apolipoprotein E isoform-dependent amyloid deposition and neuritic degeneration in a mouse model of Alzheimer's disease. Proc. Natl Acad. Sci. USA https://doi.org/10.1073/pnas.050004797 (2000).

15. Verghese, P. B. et al. ApoE influences amyloid-beta (Abeta) clearance despite minimal apoE/Abeta association in physiological conditions. Proc. Natl Acad. Sci. USA 110, E1807-E1816 (2013).

16. Hansen, D. V., Hanson, J. E. \& Sheng, M. Microglia in Alzheimer's disease. J. Cell Biol. 217, 459-472 (2018)

17. Deczkowska, A. et al. Disease-associated microglia: a universal immune sensor of neurodegeneration. Cell 173, 1073-1081 (2018).

18. Selkoe, D. J. \& Hardy, J. The amyloid hypothesis of Alzheimer's disease at 25 years. EMBO Mol. Med. 8, 595-608 (2016)

19. Keren-Shaul, H. et al. A unique microglia type associated with restricting development of Alzheimer's disease. Cell 169, 1276-1290.e1217 (2017).

20. Atagi, Y. et al. Apolipoprotein E is a ligand for triggering receptor expressed on myeloid cells 2 (TREM2). J. Biol. Chem. 290, 26043-26050 (2015).

21. Yeh, F. L., Wang, Y., Tom, I., Gonzalez, L. C. \& Sheng, M. TREM2 binds to apolipoproteins, including APOE and CLU/APOJ, and thereby facilitates uptake of amyloid-beta by microglia. Neuron 91, 328-340 (2016)

22. Song, W. et al. Alzheimer's disease-associated TREM2 variants exhibit either decreased or increased ligand-dependent activation. Alzheimers Dement. 13, 381-387 (2017).

23. Yuan, P. et al. TREM2 haplodeficiency in mice and humans impairs the microglia barrier function leading to decreased amyloid compaction and severe axonal dystrophy. Neuron 90, 724-739 (2016)

24. Fitz, N. F. et al. Trem 2 deficiency differentially affects phenotype and transcriptome of human APOE3 and APOE4 mice. Mol. Neurodegener. 15, 41 (2020).

25. Wahrle, S. E. et al. ABCAl is required for normal central nervous system ApoE levels and for lipidation of astrocyte-secreted apoE. J. Biol. Chem. 279, 40987-40993 (2004)

26. Duong, P. T., Weibel, G. L., Lund-Katz, S., Rothblat, G. H. \& Phillips, M. C. Characterization and properties of pre beta-HDL particles formed by ABCA1-mediated cellular lipid efflux to apoA-I. J. Lipid Res. 49, 1006-1014 (2008)

27. Fitz, N. F. et al. ABCA1 deficiency affects basal cognitive deficits and dendritic density in mice. J. Alzheimers Dis. 56, 1075-1085 (2017).

28. Lesné, S. et al. A specific amyloid-beta protein assembly in the brain impairs memory. Nature 440, 352-357 (2006).

29. Lefterov, I. et al. Apolipoprotein A-I deficiency increases cerebral amyloid angiopathy and cognitive deficits in APP/PS1DeltaE9 mice. J. Biol. Chem. 285, 36945-36957 (2010)

30. Lee, E. et al. Single-nucleotide polymorphisms are associated with cognitive decline at Alzheimer's disease conversion within mild cognitive impairment patients. Alzheimers Dement. 8, 86-95 (2017)

31. Yu, F. X., Johnston, P. A., Sudhof, T. C. \& Yin, H. L. gCap39, a calcium ionand polyphosphoinositide-regulated actin capping protein. Science $\mathbf{2 5 0}$ 1413-1415 (1990).

32. Galatro, T. F. et al. Transcriptomic analysis of purified human cortical microglia reveals age-associated changes. Nat. Neurosci. 20, 1162-1171 (2017).

33. Hammond, T. R. et al. Single-cell RNA sequencing of microglia throughout the mouse lifespan and in the injured brain reveals complex cell-state changes. Immunity 50, 253-271 e256 (2019).

34. Wang, Y. et al. TREM2 lipid sensing sustains the microglial response in an Alzheimer's disease model. Cell 160, 1061-1071 (2015)
35. Chausse, B., Kakimoto, P. A. \& Kann, O. Microglia and lipids: how metabolism controls brain innate immunity. Semin. Cell Dev. Biol. https://doi. org/10.1016/j.semcdb.2020.08.001 (2020).

36. Kosicek, M. \& Hecimovic, S. Phospholipids and Alzheimer's disease: alterations, mechanisms and potential biomarkers. Int. J. Mol. Sci. 14, 1310-1322 (2013).

37. Shi, Y. \& Holtzman, D. M. Interplay between innate immunity and Alzheimer disease: APOE and TREM2 in the spotlight. Nat. Rev. Immunol. 18, 759-772 (2018).

38. Wisniewski, T. \& Drummond, E. APOE-amyloid interaction: therapeutic targets. Neurobiol. Dis. 138, 104784 (2020).

39. Kanekiyo, T., Xu, H. \& Bu, G. ApoE and Abeta in Alzheimer's disease: accidental encounters or partners? Neuron 81, 740-754 (2014).

40. Nguyen, A. T. et al. APOE and TREM2 regulate amyloid-responsive microglia in Alzheimer's disease. Acta Neuropathol. 140, 477-493 (2020).

41. Krasemann, S. et al. The TREM2-APOE pathway drives the transcriptional phenotype of dysfunctional microglia in neurodegenerative diseases. Immunity 47, 566-581 e569 (2017).

42. Song, W. M. et al. Humanized TREM 2 mice reveal microglia-intrinsic and -extrinsic effects of R47H polymorphism. J. Exp. Med. 215, 745-760 (2018).

43. Sala Frigerio, C. et al. The major risk factors for Alzheimer's Disease: age, sex and genes modulate the microglia response to Abeta plaques. Cell Rep. 27, 1293-1306 e1296 (2019).

44. Fitz, N. F. et al. Liver X receptor agonist treatment ameliorates amyloid pathology and memory deficits caused by high-fat diet in APP23 mice. J. Neurosci.30, 6862-6872 (2010).

45. Lefterov, I. et al. RNA-sequencing reveals transcriptional up-regulation of Trem 2 in response to bexarotene treatment. Neurobiol. Dis. 82, 132-140 (2015)

46. Aviram, R. et al. Lipidomics analyses reveal temporal and spatial lipid organization and uncover daily oscillations in intracellular organelles. Mol. Cell 62, 636-648 (2016)

47. Han, X., Yang, K. \& Gross, R. W. Multi-dimensional mass spectrometry-based shotgun lipidomics and novel strategies for lipidomic analyses. Mass Spectrom. Rev. 31, 134-178 (2012).

48. Nam, K. N. et al. Effect of high fat diet on phenotype, brain transcriptome and lipidome in Alzheimer's model mice. Sci. Rep. 7, 4307 (2017).

49. Yang, K., Cheng, H., Gross, R. W. \& Han, X. Automated lipid identification and quantification by multidimensional mass spectrometry-based shotgun lipidomics. Anal. Chem. 81, 4356-4368 (2009).

50. Tyurina, Y. Y. et al. Characterization of cardiolipins and their oxidation products by LC-MS analysis. Chem. Phys. Lipids 179, 3-10 (2014).

51. Marsh, S. E. et al. The adaptive immune system restrains Alzheimer's disease pathogenesis by modulating microglial function. Proc. Natl Acad. Sci. USA 113, E1316-E1325 (2016).

52. Nam, K. N. et al. Integrated approach reveals diet, APOE genotype and sex affect immune response in APP mice. Biochim. Biophys. Acta Mol. Basis Dis. 1864, 152-161 (2018)

53. Butler, A., Hoffman, P., Smibert, P., Papalexi, E. \& Satija, R. Integrating singlecell transcriptomic data across different conditions, technologies, and species. Nat. Biotechnol. 36, 411-420 (2018).

54. Street, K. et al. Slingshot: cell lineage and pseudotime inference for single-cell transcriptomics. BMC Genomics 19, 477 (2018)

55. Iordanova, B., Vazquez, A., Kozai, T. D., Fukuda, M. \& Kim, S. G. Optogenetic investigation of the variable neurovascular coupling along the interhemispheric circuits. J. Cereb. Blood Flow. Metab. 38, 627-640 (2018).

\section{Acknowledgements}

This research was supported by the National institute of Health, grant number AG056371, AG057565, AG066198 (I.L. and R.K), NS116450 (B.E.I.), and NS094396 (T. D.Y.K.) and the Alzheimer's Association, 2018-AARG-590509 (N.F.F.) and AARF-16443213 (K.N.N.). Human brain samples were provided by ADRC Pittsburgh (P30AG066468) and Dr. Peter Nelson, University of Kentucky ADRC (P30 AG028383) We thank all members of Lefterov/Koldamova lab for their excellent technical assistance.

\section{Author contributions}

N.F.F., I.L., and R.K. conceived and designed the research and wrote the manuscript. All authors edited the drafts. N.F.F. and B.E.P. set up and performed mouse experiments, IHC, and A $\beta$ aggregation experiments. C.M.W. and K.N.N. isolated APOE lipoproteins from ACM. K.N.N. performed, analyzed, and wrote the single cell RNA sequencing experiments, and in vitro experiments. C.M.W. and F.L. performed flow cytometry and bulk sequencing experiments and analysis. C.M.W. performed FISH experiments. R.J.B. participated in the analysis of the single cell RNA sequencing data. B.E.I. performed, analyzed, and wrote the two-photon imaging experiments. V.E.K., Y.Y.T., and X.H. performed lipidomics experiments and analysis. T.D.Y.K. provided essential resources.

\section{Competing interests}

The authors declare no competing interests. 


\section{Additional information}

Supplementary information The online version contains supplementary material available at https://doi.org/10.1038/s41467-021-23762-0.

Correspondence and requests for materials should be addressed to I.L. or R.K.

Peer review information Nature Communications thanks the anonymous reviewer(s) for their contribution to the peer review of this work. Peer reviewer reports are available.

Reprints and permission information is available at http://www.nature.com/reprints

Publisher's note Springer Nature remains neutral with regard to jurisdictional claims in published maps and institutional affiliations. (c) (P) Open Access This article is licensed under a Creative Commons Attribution 4.0 International License, which permits use, sharing, adaptation, distribution and reproduction in any medium or format, as long as you give appropriate credit to the original author(s) and the source, provide a link to the Creative Commons license, and indicate if changes were made. The images or other third party material in this article are included in the article's Creative Commons license, unless indicated otherwise in a credit line to the material. If material is not included in the article's Creative Commons license and your intended use is not permitted by statutory regulation or exceeds the permitted use, you will need to obtain permission directly from the copyright holder. To view a copy of this license, visit http://creativecommons.org/ licenses/by/4.0/.

(C) The Author(s) 2021 